\title{
Morse and Melnikov Functions for NLS Pde's
}

\author{
Y. Li ${ }^{1}$, David W. McLaughlin ${ }^{2, \star}$ \\ 1 Department of Mathematics, Princeton University, \\ Princeton, NJ 08544, USA \\ 2 Department of Mathematics, Program in Applied and Computational Mathematics, \\ Princeton University, Princeton, NJ 08544, USA
}

Received: June 5, 1992/in revised form: February 25, 1993

\begin{abstract}
The theory of the focusing NLS equation under periodic boundary conditions, together with the Floquet spectral theory of its associated Zakharov-Shabat linear operator $\hat{L}$, is developed in sufficient detail for later use in studies of perturbations of the NLS equation. "Counting lemmas" for the non-selfadjoint operator $\hat{L}$, are established which control its spectrum and show that all of its eccentricities are finite in number and must reside within a finite disc $D$ in the complex eigenvalue plane. The radius of the disc $D$ is controlled by the $H^{1}$ norm of the potential $\vec{q}$. For this integrable NLS Hamiltonian system, unstable tori are identified, and Backlund transformations are then used to construct global representations of their stable and unstable manifolds - "whiskered tori" for the NLS pde.

The Floquet discriminant $\Delta(\lambda ; \vec{q})$ is used to introduce a natural sequence of NLS constants of motion, $\left[\mathbf{F}_{j}(\vec{q}) \equiv \Delta\left(\lambda=\lambda_{j}^{c}(\vec{q}) ; \vec{q}\right)\right.$, where $\lambda_{j}^{c}$ denotes the $j^{\text {th }}$ critical point of the Floquet discriminant $\Delta(\lambda)$ ]. A Taylor series expansion of the constants $\mathbf{F}_{j}(\vec{q})$, with explicit representations of the first and second variations, is then used to study neighborhoods of the whiskered tori. In particular, critical tori with hyperbolic structure are identified through the first and second variations of $\mathbf{F}_{j}(\vec{q})$, which themselves are expressed in terms of quadratic products of eigenfunctions of $\hat{L}$. The second variation permits identification, within the disc $D$, of important bifurcations in the spectral configurations of the operator $\hat{L}$. The constant $\mathbf{F}_{j}(\vec{q})$, as the height of the Floquet discriminant over the critical point $\lambda_{j}^{c}$, admits a natural interpretation as a Morse function for NLS isospectral level sets. This Morse interpretation is studied in some detail. It is valid globally for the infinite tail, $\left\{\mathbf{F}_{j}(\vec{q})\right\}_{|j|>N}$, which is associated with critical points outside the disc $D$. Within this disc, the interpretation is only valid locally, with the same obstruction to its global validity as to a global ordering of the spectrum. Nevertheless, this local Morse theory, together with the Backlund representations of the whiskered tori, produces extremely clear pictures of the stratification of NLS invariant sets near these whiskered tori - pictures which are
\end{abstract}

* Funded in part by AFOSR-90-0161 and by NSF DMS 8922717 A01 
useful in the study of perturbations of NLS. Finally, a natural connection is noted between the constants $\mathbf{F}_{j}(\vec{q})$ of the integrable theory and Melnikov functions for the theory of perturbations of the NLS equation. This connection generates a simple, but general, representations of the Melnikov functions.

\section{Contents}

1. Introduction . . . . . . . . . . . . . . . . . . . . . . . . 176

2. The General Setting . . . . . . . . . . . . . . . . . . . 178

2.1. NLS as a Hamiltonian System . . . . . . . . . . . . . . . . . . 178

2.2. Spectral Background … . . . . . . . . . . . . . . . . . . . . . . . . . . . . . . . . 179

2.2.1 Floquet Spectral Theory . . . . . . . . . . . . . . . . . . . . . . 180

2.3. Counting Lemmas . . . . . . . . . . . . . . . . . . . . . . . . 182

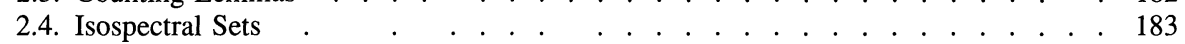

2.5. An Ordering of the Spectrum . . . . . . . . . . . . . . . . . . . . . . . . . 184

2.6. The First Variations of $\Delta(\lambda ; \vec{q}) \quad$. . . . . . . . . . . . . . . . . . . . . . 187

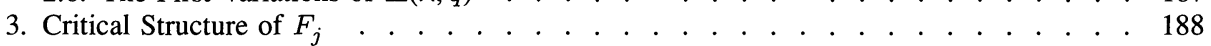

3.1. First Variational Condition . . . . . . . . . . . . . . . . . . . . . . . . . . . . 188

3.2. Critical Tori . . . . . . . . . . . . . . . . . . . . . . . . . . . . . . . . 190

3.3. The Hessian of $F_{j}$. . . . . . . . . . . . . . . . . . . . . . . . . . . . . . . . . . . 190

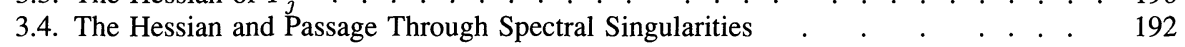

4. Global Representations of Whiskered Tori . . . . . . . . . . . . . . . . . . . 194

4.1. Linearized Instabilities . . . . . . . . . . . . . . . . . . . . . . . 194

4.2. Bäcklund Transformations . . . . . . . . . . . . . . . . . . . . . . 195

4.3. An Example: The Spatially Uniform Plane Wave . . . . . . . . . . . . . . . . . . 196

5. Toward a Morse Description of the Isospectral Stratification . . . . . . . . . . . . . . 197

5.1. Outside the Disc $D:|j|>N$. . . . . . . . . . . . . . . . . . . . . . . . . . . . 199

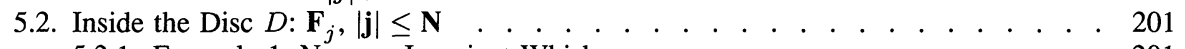

5.2.1. Example 1: Near an Invariant Whisker . . . . . . . . . . . . . . . 201

5.2.2. Even Functions . . . . . . . . . . . . . . . . . . . . . . . . . . . . . . 202

5.2.3. No Spatial Symmetry

5.2.4 Higher Order Critical Points . . . . . . . . . . . . . . . . . . . . . . . . . . . . . 208

5.3. Discussion . . . . . . . . . . . . . . . . . . . . . . . . . 208

6. A Melnikov Vector . . . . . . . . . . . . . . . . . . . . . . . 209

7. Conclusion . . . . . . . . . . . . . . . . . . . . . 211

\section{Introduction}

In our earlier studies [27] of damped and driven nonlinear Schrödinger (NLS) equations under periodic boundary conditions, we have observed chaotic temporal behavior which is particularly apparent when the nearby unperturbed integrable NLS equation has critical tori with hyperbolic (saddle-like) structure. In this paper we study mathematical properties of the hyperbolic structure for the integrable NLS equation, in the focusing case under periodic boundary conditions. These properties are prerequisites for any study of perturbations of the integrable NLS system.

In any integrable Hamiltonian system, hyperbolic structure consists in critical tori, called "whiskered tori" [1], whose stable and unstable manifolds comprise the "whiskers." At issue here in the infinite dimensional NLS setting is to identify, to represent, and to understand these whiskered tori, as well as their neighborhoods in function space. We accomplish these goals through the inverse spectral representation for the NLS equation with its associated linear operator $\hat{L}$. In the focusing case, this operator $\hat{L}$ is not self-adjoint. The nonselfadjointness of this second order differential 
operator $\hat{L}$ is responsible for the existence of hyperbolic structure in the focusing NLS system. Certain critical configurations in the complex spectrum of $\hat{L}$ identify whiskered tori. (In fact, the presence of hyperbolic structure in the experiments [27] on perturbed NLS is monitored numerically by following passage through these critical spectral configurations.)

To understand this nonselfadjoint spectral problem, we begin with three basic "counting lemmas" which we use to control the spectrum of $\hat{L}$. These counting lemmas identify a finite disc $D$ in the eigenvalue plane, whose radius is controlled in terms of the unstable $H^{1}$ norm of the coefficient $\vec{q}$, which in turns is controlled by the first two NLS invariants - the $L^{2}$ norm and the energy. All eccentricities (such as the critical spectral configurations) due to nonselfadjointness of $\hat{L}$ occur within the disc $D$. The counting lemmas also enable us to order the spectrum of the linear operator (locally, near a fixed $\vec{q} \in H^{1}$ ) and to use this ordering to label a basis of $L^{2}$ built from squared eigenfunction solutions of the linearization of NLS. Bäcklund, or Darboux, transformations (also built from quadratic products of eigenfunctions) are then used at the critical spectral configurations to construct global representations of the whiskered tori.

Integrable theory focuses attention upon level sets of constants of motion. In this NLS framework, these level sets are isospectral sets for the operator $\hat{L}$, and can be defined in terms of the Floquet discriminant (which generates all NLS constants of motion):

$$
\mathscr{M}(\vec{q}) \equiv\{\vec{r} \in \mathscr{F} \mid \Delta(\lambda ; \vec{r})=\Delta(\lambda ; \vec{q}) \forall \lambda \in \mathbf{C}\}
$$

A global theory of these NLS level sets is not yet complete in the nonselfadjoint case; nevertheless, it is generally believed that the level set $\mathscr{O C}$ is generically an (infinite) product of circles.

To understand the critical NLS tori and their neighborhoods in function space, as well as the associated neighborhoods of the critical spectral configurations, we interplay the compex $\lambda$ dependence of the Floquet discriminant $\Delta(\lambda ; \vec{q})$ with its dependence upon the function $\vec{q}(x)$. In terms of $\Delta(\lambda ; \vec{q})$ we introduce a natural sequence of NLS constants of motion, $\mathbf{F}_{\jmath}(\vec{q}) \equiv \Delta\left(\lambda_{j}^{c}(\vec{q}) ; \vec{q}\right)$, where $\lambda_{j}^{c}$ denotes the $j^{\text {th }}$ critical point of the Floquet discriminant $\Delta(\lambda)$. We then use a Taylor series expansion (in $\vec{q}$ ) of the constants $\mathbf{F}_{j}(\vec{q})$ to study neighborhoods of the whiskered tori. The first and second variations of $\mathbf{F}_{j}(\vec{q})$ admit concrete representations in terms of quadratic products of eigenfunctions of $\hat{L}$. The critical tori are critical functions of $\mathbf{F}_{j}(\vec{q})$ at which, for at least one $j$, the first variation vanishes. When the first variation vanishes at a critical torus, the second variation, or Hessian, can be indefinite, which leads to the identification, within the disc $D$, of important bifurcations between "cross" and "gap" spectral configurations for the operator $\hat{L}$, as well as to closely related bifurcations of the NLS invariant sets.

This use of the family of constants $\left\{\mathbf{F}_{j} \forall j\right\}$ to identify critical level sets and their neighborhoods is the first step toward a "Morse theory of NLS level sets." A successful Morse theory would describe how these tori change with the (values of) the spectrum, and how they stratify (or fill out) the function space $\mathscr{F}$. Clearly, such a stratification by tori will be organized by neighborhoods of critical tori for which one or more of the circles in the infinite product has pinched off. These are exactly the critical functions of the constants $\left\{\mathbf{F}_{\jmath}(\vec{q})\right\}$.

The constant $\mathbf{F}_{\jmath}(\vec{q})$, as the height of the Floquet discriminant over the critical point $\lambda_{\jmath}^{c}$, admits a natural interpretation as a Morse function for NLS level sets. In Sect. 5 
this Morse interpretation is investigated in some detail. It is valid globally for the infinite tail $\left\{\mathbf{F}_{j}(\vec{q})\right\}_{|j|>N}$, which is associated with critical points outside the disc $D$. Within this disc, the interpretation is only valid locally, with the same obstruction to global validity as to a global ordering of the spectrum. Nevertheless, within the disc $D$ we present explicit examples of this local viewpoint, which together with the Bäcklund representations of the whiskered tori, produces extremely clear pictures of the stratification of NLS invariant sets near these whiskered tori. These examples are more than adequate for our study of perturbations of NLS. We hope they will convince others to complete a global Morse theory of NLS level sets.

Finally, in Sect. 6, we show that the constants $\mathbf{F}_{\text {}}$ of the integrable theory also generate natural Melnikov functions for the theory of perturbations of NLS, precisely because these constants are critical on the critical tori. In addition, we derive explicit representations of the Melnikov function in terms of quadratic products of eigenfunctions.

This work is a continuation of the initial study of $[7,8]$ for the sine-Gordon equation, and of the note [9]. However, it differs substantially from those earlier studies in that the role of finite genus algebraic geometry is downplayed and replaced by the functional analysis framework of [29]. Specifically, the explicit use of the counting lemmas to control the nonselfadjoint spectral theory of $\hat{L}$ is new, as are the explicit Hessian calculations. The detailed Morse study is new; in particular, the obstruction to its global validity, the Bott function $\mathbf{G}$, the behavior near an arbitrary complex double point where several Bott functions must be studied simultaneously, and the pictures of the level sets in a six dimensional example were not known previously.

For the spectral theory, we follow closely the work, in references [29, 14], extending it to the non-self-adjoint case in order to treat hyperbolic structure.

Finally, the table of contents serves as an adequate outline for the paper.

\section{The General Setting}

\subsection{NLS as a Hamiltonian System}

We consider the focusing nonlinear Schrödinger equation (NLS)

$$
-i q_{t}+q_{x x}+2|q|^{2} q=0
$$

with periodic boundary conditions $q(x+1)=q(x)$. Energy arguments, using "higher" constants of motion, establish existence in $H^{k}, k>1$.

To view NLS as an (infinite dimensional) dynamical system, we first fix the phase space. We begin with the ambient space of $H_{\text {per }}^{1}\left([0,1] ; C^{2}\right) \subset L_{\text {loc }}^{2}\left(R, C^{2}\right)$ of periodic, complex valued two vector functions of $x$, which are square integrable over $[0,1]$ with square integrable first derivative. It will be convenient to fix the following real Hilbert spaces:

$$
\mathscr{S}^{ \pm} \equiv\left\{(q, r) \in H_{\mathrm{per}}^{1} \mid r= \pm \bar{q}\right\}
$$

For focusing NLS, the phase space is then the embedding of $C^{\infty}$ in $\mathscr{S}^{-}$,

$$
\mathscr{F} \equiv C^{\infty} \subset \mathscr{S}^{-} \text {. }
$$

On this phase space, NLS is a Hamiltonian system,

$$
-i \vec{q}_{t}=J \operatorname{grad} H \text {. }
$$


Here $J$ denotes the matrix

$$
J=\left(\begin{array}{cc}
0 & 1 \\
-1 & 0
\end{array}\right)
$$

and the Hamiltonian $H: \mathscr{F} \rightarrow R$ is given by

$$
H(\vec{q}) \equiv \int_{0}^{1}\left[q_{x} r_{x}+q^{2} r^{2}\right] d x .
$$

The complete integrability of the NLS Hamiltonian system is established through the "inverse spectral transform" which we now describe.

\subsection{Spectral Background}

The NLS equation can be integrated with the Zakharov-Shabat linear system [34]

$$
\begin{aligned}
\varphi_{x} & =U^{(\lambda)} \varphi, \\
\varphi_{t} & =V^{(\lambda)} \varphi,
\end{aligned}
$$

where

$$
\begin{gathered}
U^{(\lambda)}=i \lambda \sigma_{3}+i\left(\begin{array}{cc}
0 & q \\
-r & 0
\end{array}\right) ; \\
V^{(\lambda)}=2 i \lambda^{2} \sigma_{3}+i q r \sigma_{3}+\left(\begin{array}{cc}
0 & 2 i \lambda q+q_{x} \\
-2 i \lambda r+r_{x} & 0
\end{array}\right),
\end{gathered}
$$

where $\sigma_{3}$ denotes the third Pauli matrix $\sigma_{3}=\operatorname{diag}(1,-1)$. Compatibility of the over determined system $(2.2,2.3)$ insures that the coefficient $q$ satisfies the NLS equation.

Focusing attention upon the "spatial flow" (2.2), we let $Y^{(1)}, Y^{(2)}$ be the fundamental solutions of ode (2.2), i.e. solutions with the initial condition:

$$
Y^{(1)}(0)=\left(\begin{array}{l}
1 \\
0
\end{array}\right), \quad Y^{(2)}(0)=\left(\begin{array}{l}
0 \\
1
\end{array}\right)
$$

With these initial conditions, the differential equation may be rewritten as an integral equation

$$
\begin{gathered}
Y^{(1)}=\left[\begin{array}{c}
e^{i \lambda x} \\
0
\end{array}\right]+\int_{0}^{x} \exp \left[i \sigma_{3} \lambda(x-t)\right]\left[\begin{array}{cc}
0 & i q \\
-i r & 0
\end{array}\right] Y^{(1)} d t, \\
Y^{(2)}=\left[\begin{array}{c}
0 \\
e^{-i \lambda x}
\end{array}\right]+\int_{0}^{x} \exp \left[i \sigma_{3} \lambda(x-t)\right]\left[\begin{array}{cc}
0 & i q \\
-i r & 0
\end{array}\right] Y^{(2)} d t,
\end{gathered}
$$

which, through Weyl's iteration procedure, produces formal series representations of $Y^{(\jmath)}, j=1,2$, together with the estimates

$$
\begin{aligned}
& \left|Y^{(1)}\right| \leq \exp \{|\operatorname{Im} \lambda| x\}\left[\begin{array}{l}
\cosh \left(\|\vec{q}\|_{2} \sqrt{x / 2}\right) \\
\sinh \left(\|\vec{q}\|_{2} \sqrt{x / 2}\right)
\end{array}\right], \\
& \left|Y^{(2)}\right| \leq \exp \{|\operatorname{Im} \lambda| x\}\left[\begin{array}{l}
\sinh \left(\|\vec{q}\|_{2} \sqrt{x / 2}\right) \\
\cosh \left(\|\vec{q}\|_{2} \sqrt{x / 2}\right)
\end{array}\right] .
\end{aligned}
$$

Precisely, one has the following 
Theorem 1. The formal power series for $Y^{(1)}$ and $Y^{(2)}$ converge uniformly on bounded subsets of $[0,1] \times C \times L_{\mathbf{C}}^{2} \times L_{\mathbf{C}}^{2}$. Moreover, they satisfy the integral equations (2.4) and (2.5), and the estimate (2.6) and (2.7). For each $x \in[0,1], Y^{(1)}$ and $Y^{(2)}$ are entire functions on $C \times L_{\mathbf{C}}^{2} \times L_{\mathbf{C}}^{2}$. The solution $Y^{(\jmath)}(\cdot ; \lambda ; \vec{q}), j=1,2$, is analytic as a map from $C \times L_{\mathbf{C}}^{2} \times L_{\mathbf{C}}^{2}$ into $H^{1}$.

2.21 Floquet Spectral Theory. The integration of the NLS equation is accomplished through the spectral theory of the differential operator $\hat{L}=\hat{L}(\vec{q})$,

$$
\hat{L}=-i \sigma_{3} \frac{d}{d x}-\left(\begin{array}{cc}
0 & q \\
r & 0
\end{array}\right),
$$

for coefficients $\vec{q}=(q, r)$ which are periodic, $H^{1}$ functions of $x$. We view $\hat{L}$ as an operator on $L^{2}(\mathbf{R})$, with dense domain $H^{1}$. In this $L^{2}$ setting, the spectrum $\sigma(\hat{L})$ is defined as the closure of the set of complex $\lambda$ for which there exists a solution of

$$
\hat{L} \psi=\lambda \psi
$$

which is bounded for all $x \in(-\infty,+\infty)$. Since the coefficient $\vec{q}$ is a periodic function of $x$, Floquet theory can be used to characterize this spectrum.

Floquet theory begins from the fundamental matrix $M=M(x ; \lambda ; \vec{q})$, which is defined in terms of $Y^{(1)}$ and $Y^{(2)}$ :

$$
M=\text { columns }\left\{Y^{(1)}, Y^{(2)}\right\} .
$$

Next, one introduces the transfer matrix $T$

$$
T(\lambda ; \vec{q})=M(1 ; \lambda ; \vec{q}) .
$$

Then the spectrum $\sigma(\hat{L})$ can be characterized as the set of all $\lambda$ for which the $2 \times 2$ matrix $T$ has eigenvalues on the unit circle. Since the $\operatorname{det} T=1$, this is in turn determined by a single scalar function called the Floquet discriminant:

$$
\Delta: \mathbf{C} \times H^{1} \rightarrow \mathbf{C} \quad \text { by } \quad \Delta(\lambda ; \vec{q})=\operatorname{tr}\{T(\lambda ; \vec{q})\} .
$$

In terms of $\Delta$ the spectrum is given by

$$
\sigma(\hat{L}(\vec{q}))=\{\lambda \in \mathbf{C} \mid \Delta(\lambda) \text { is real and }-2 \leq \Delta \leq+2\} .
$$

The integration of NLS is actually accomplished through an interplay between the $\lambda$ and $\vec{q}$ dependence of the Floquet discriminant $\Delta(\lambda ; \vec{q})$. First one establishes, as a corollary to Theorem 1 , that $\Delta$ is entire in both $\lambda$ and $\vec{q}=(q, r)$. Moreover, a calculation [27] shows that its Poisson bracket with the NLS Hamiltonian vanishes:

$$
\{\Delta(\lambda ; \vec{q}), H(\vec{q})\}=0 \forall \lambda,
$$

where the Poisson bracket is defined as

$$
\{F, G\}=\int_{0}^{1}(\operatorname{grad} F, J \operatorname{grad} G) d x .
$$

Thus, $\Delta(\lambda ; \vec{q})$ generates an infinite family of NLS constants of motion, one for each $\lambda$. Moreover, this family of invariants pairwise commutes,

$$
\left\{\Delta(\lambda ; \vec{q}), \Delta\left(\lambda^{\prime} ; \vec{q}\right)\right\}=0 \forall \lambda, \lambda^{\prime} .
$$


The difficulty with the spectral theory of $\hat{L}$ is that this operator is not selfadjoint. Nevertheless, certain properties of its spectrum follow as in the standard Floquet theory of Hill's operator [22]. The spectrum occurs in bands, not necessarily real, which terminate at periodic points, $\lambda_{j}$, for which $\Delta\left(\lambda_{j}\right)= \pm 2$. Furthermore, asymptotic behavior of $\Delta(\lambda)$ for large $|\lambda|$ is easy to establish, from which one obtains the asymptotic behavior of the periodic points:

$$
\lambda_{j} \sim j \pi \quad \text { as } \quad \lambda_{j} \rightarrow \pm \infty
$$

The above spectral properties apply for general $(q, r) \in H_{\text {per }}^{1}$; however, we are primarily interested in focusing NLS which has the constraint $r=-\bar{q}$. In this focusing case, symmetries of $\hat{L}$ imply the following facts:

(i) $\mathbf{R} \subset \sigma(\hat{L})$,

(ii) $\lambda \in \sigma \Rightarrow \bar{\lambda} \in \sigma$,

(iii) for even $q(x), \lambda \in \sigma \Rightarrow-\lambda \in \sigma$.

Next we define critical points and multiple points: First, critical points are defined by the condition

$$
\left.\frac{d \Delta(\lambda ; q)}{d \lambda}\right|_{\lambda^{c}(q)}=0 ;
$$

while a multiple point, denoted $\lambda^{(m)}$, is a critical point for which

$$
\left|\Delta\left(\lambda^{c} ; q, r\right)\right|=2 .
$$

The algebraic multiplicity of $\lambda^{(m)}$ is defined as the order of the zero of $\Delta(\lambda) \pm 2$. Usually it is 2 , but it can exceed 2; when it does equal 2, we call the multiple point a double point, and denote it by $\lambda^{(d)}$. The geometric multiplicity of $\lambda^{(m)}$ is defined as the dimension of the eigenspace of $\hat{L}$ at $\lambda^{(m)}$, and is either 1 or 2 .

Turning to properties of the spectrum of $\hat{L}$ which are rather directly related to the nonselfadjointness of $\hat{L}$, we consider a critical point $\lambda^{c}$ at which

$$
\left|\Delta\left(\lambda^{c}\right)\right|<2 .
$$
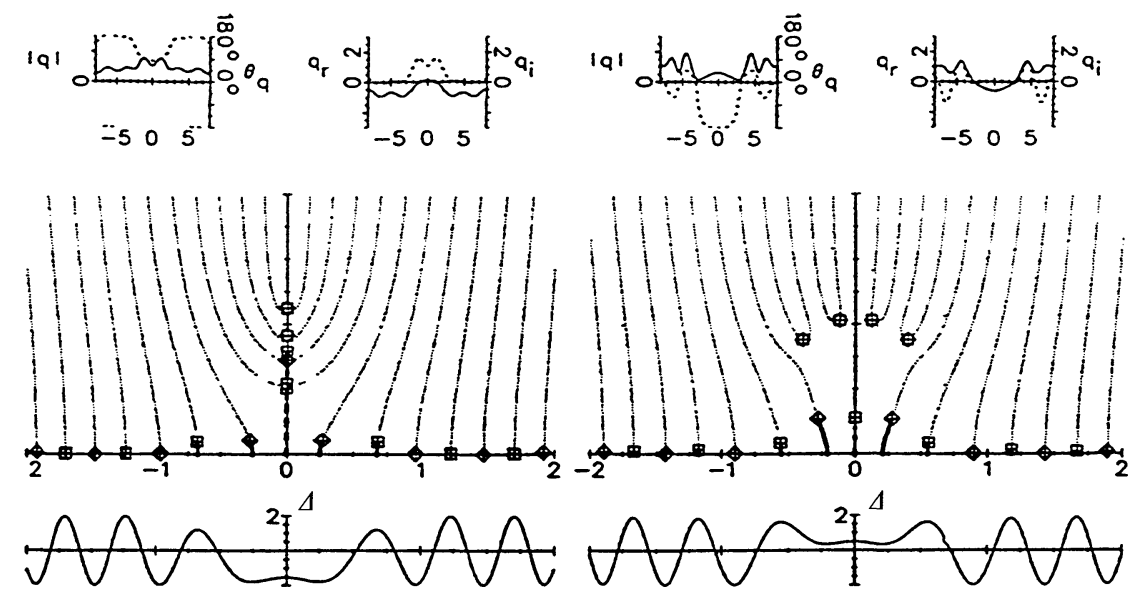

Fig. 2.1. Some typical spectral profiles (The spectrum is solid; curves of real $\Delta$ are dotted)

Such a critical point is a point of bifurcation of the spectrum. (See Fig. 2.1 for two typical spectral profiles; more may be found in [27], with detailed descriptions.) 
Furthermore, asymptotic behavior for large real $\lambda$ shows that there are a countable number of such critical points on the real axis which approach $j \pi$ as $j \rightarrow \infty$, and at which a short "spine" of spectrum bifurcates from the real axis into the complex $\lambda$ plane. In addition to these short spines of spectrum which are connected (through spectrum) to the real axis, examples related to solitons show that there can also exist curves of spectrum in the complex $\lambda$ plane which are not connected (through spectrum) to the real axis. Nevertheless, this nonselfadjoint behavior is not as complicated as one might initially anticipate. In fact, it can be controlled with the aid of certain "counting lemmas" which we now describe.

\subsection{Counting Lemmas}

Following Pöschel and Trubowitz [29] for Hill's equation, one [20] can establish counting lemmas for control of the spectrum. For convenience, we assume that $r=-\bar{q}$.

Lemma 1 (Counting Lemma for Critical Points). For $q \in H^{1}$, set $N=N\left(\|q\|_{H^{1}}\right) \in$ $\mathbf{Z}^{+}$by

$$
N\left(\|q\|_{H^{1}}\right)=2\left[\|q\|_{2}^{2} \cosh \left(\|q\|_{2}\right)+3\|q\|_{H^{1}} \sinh \left(\|q\|_{2}\right)\right],
$$

where $[z] \equiv$ first integer greater than $x$. Consider

$$
\Delta^{\prime}(\lambda ; q)=\frac{d}{d \lambda} \Delta(\lambda ; q)
$$

\section{Then}

- (i) $\Delta^{\prime}(\lambda ; q)$ has exactly $2 N+1$ zeros (counted according to multiplicity) in the interior of the disc $D \equiv\{\lambda \in \mathbf{C}:|\lambda|<(2 N+1) \pi / 2\}$;

- (ii) $\forall k \in \mathbf{Z},|k|<N, \Delta^{\prime}(\lambda, q)$ has exactly one zero in each disc $\{\lambda \in \mathbf{C}:|\lambda-k \pi|<$ $\pi / 4\}$.

- (iii) $\Delta^{\prime}(\lambda ; q)$ has no other zeros

- (iv) For $|\lambda|>(2 N+1) \pi / 2$, the zeros of $\Delta^{\prime},\left\{\lambda_{j}^{c},|j|>N\right\}$, are all real, simple, and satisfy the asymptotics

$$
\lambda_{j}^{c}=j \pi+o(1) \quad \text { as } \quad|j| \rightarrow \infty .
$$

Similar counting lemmas exist for the periodic points and for the Dirichlet eigenvalues [27]. The proof [20] of these counting lemmas proceeds as is in [29]. Rather than present it here, we make several remarks:

Remark 1. To understand the content of these lemmas, it is useful to begin with a consideration of the spectrum for $q=0$, followed with the spectrum for $q(x)=c$, a constant independent of $x$. In both of these cases the spectrum can be computed explicitly. The spectrum for a general potential approaches that for $q=0$ as $\lambda \rightarrow \infty$, as is easy to establish through asymptotic expansions. As a result, the critical points $\lambda_{j}^{c}$ must approach $j \pi$ for large $j$. The counting lemmas provide somewhat different information than this asymptotic behavior. Rather than demanding that the critical point $\lambda_{j}^{c}$ be extremely close to $j \pi$, one relaxes the tolerance and only demands that $\lambda_{j}^{c}$ be within a disc of radius $\pi / 4$ centered at $j \pi$ (see Fig. 2.2). With this relaxed tolerance, the desired behavior sets in at much smaller $j,|j|>N$. 


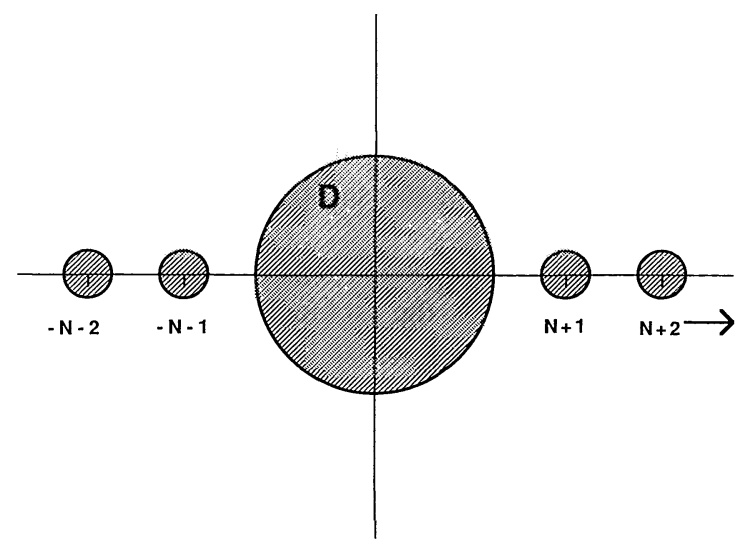

Fig. 2.2. The disc $D$ and the integer $N$ of the Counting Lemmas

Remark 2. Moreover, the lemmas state that this integer $N$ is controlled by the $H^{1}$ norm of $\vec{q}$. In the case of Hill's equation, similar control can be obtained by only the $L^{2}$ norm. For the Zakharov-Shabat system, such $L^{2}$ control is not possible, as can be seen from a counter example which is built from the potential $q^{(k)}=q \exp (i k x)$, $k \in \mathbf{R}$. (The spectrum of $q^{(k)}$ is shifted by $k / 2$ from the spectrum of $q$, yet $q$ and $q^{(k)}$ have the same $L^{2}$ norm. Their $H^{1}$ norms differ, of course.)

Remark 3. From these two counting lemmas, one obtains very good control on the spectrum outside a disc $D$ of radius $(2 N+1) \pi / 2$, together with some control of the integer $N$. Within the disc $D$, little is known about possible spectral configurations; however, the problem of classifying all spectral configurations has been reduced to a finite counting problem - possibly a very difficult one. Nevertheless, all eccentric behavior due to the nonselfadjoint nature of $\hat{L}$ is restricted to the interior of this finite disc.

Remark 4. In the counting lemmas, the integer $N$ is controlled by the $H^{1}$ norm of $\vec{q}$. While this may be useful for controlling the behavior of perturbations of NLS, it is not an NLS invariant. Sobolev estimates can be used to replace the $H^{1}$ control by control in terms of the first two invariants.

Remark 5. These counting lemmas amount to an extension of the Gersch-Goren theorems of matrix linear algebra [33] to the (infinite dimensional) setting of ordinary differential equations [28]. In the matrix setting, the bounds are optimal in the sense that matrices can be constructed which realize the bounds. It would be interesting to construct such realizations in the differential operator case [16].

\subsection{Isospectral Sets}

Since the Floquet discriminant $\Delta$ is an NLS constant of the motion, any spectral information obtained from it, such as that discussed above, is necessarily "action" information. The values of these action variables fix a particular level set,

$$
\mathscr{l}(\vec{q}) \equiv\{\vec{r} \in \mathscr{F} \mid \Delta(\lambda ; \vec{q})=\Delta(\lambda ; \vec{q}) \forall \lambda \in \mathbf{C}\}
$$


For focusing NLS with nonselfadjoint $\hat{L}$, a global description of $\mathscr{C}(\vec{q})$ is not yet available. (This is in contrast to the $\mathrm{KdV}$ situation [24] with its selfadjoint $\hat{L}$.) Locally, in a sufficiently small neighborhood of an " $N$-phase" $\vec{q}_{N}, \mathscr{M l}(\vec{q})$ is an infinite dimensional torus $(\ldots \times S \times S \times S \times \ldots)$; it can be singular, with one or more of the circles pinched away. (See [23] for the similar local behavior in the sine-Gordon case.)

Given an isospectral level set $\mathscr{l}(\vec{q})$, one seeks coordinates ("angles") for it. Such angle information is provided (modulo the problems of "reality constraints" [12]; [6]) by Dirichlet eigenvalues, which are defined as the zeros of $\tilde{M}_{21}$ :

$$
\left.\tilde{M}_{21}\right|_{\lambda=\mu_{\jmath}(q)}=0 \text {, }
$$

where $\tilde{M}$ is a unitary transformation of the fundamental matrix $M$,

$$
\tilde{M}=U M U^{\dagger}, \quad U=\frac{1}{\sqrt{2}}\left(\begin{array}{cc}
1 & -1 \\
i & i
\end{array}\right) .
$$

These Dirichlet eigenvalues $\mu_{j}$ are not NLS invariants; rather, they execute oscillatory motion under the NLS flow. As indicated by the following "trace formulas," Dirichlet eigenvalues provide the additional "angle" information, which together with the periodic eigenvalues, provide a representation of $q(x)$ :

$$
\begin{gathered}
q(x)+r(x)=-\sum_{k \in Z}\left[\lambda_{2 k}+\lambda_{2 k-1}-2 \mu_{k}(x)\right], \\
q(x)-r(x)=i \sum_{k \in Z}\left[\lambda_{2 k}+\lambda_{2 k-1}-2 \nu_{k}(x)\right]
\end{gathered}
$$

Here $\nu_{\jmath}$ are the Dirichlet eigenvalues for $i q$,

$$
\left.\tilde{M}_{21}(1 ; \lambda ; i q)\right|_{\lambda=\nu_{\jmath}}=0 \text {, }
$$

and $\left(\mu_{j}(x), \nu_{j}(x)\right)$ denote eigenvalues for the translated coefficient $q^{(x)}\left(x^{\prime}\right)=q\left(x^{\prime}+x\right)$.

The counting lemma for the Dirichlet eigenvalues shows that these eigenvalues can be placed in a natural one-to-one correspondence with the critical points. In particular, for each $j$ greater than the " $N$ " of the counting lemmas, there exists exactly one $\mu_{j}$ near the $j^{\text {th }}$ (real) critical point $\lambda_{j}^{c}$; with exactly $2 N+1 \mu$ 's inside the disc of radius $(2 N+1) \pi / 2$. Moreover, symmetries of $M$ show that at each real double point $\lambda^{d}$ (a critical point at which $\Delta^{2}-4$ vanishes), the associated Dirichlet eigenvalue must be locked to that double point. On the other hand, at a complex double point [which is necessarily within the disc of radius $(2 N+1) \pi / 2]$, the associated Dirichlet eigenvalue may either be locked or it may be free to move. This property of complex Dirichlet eigenvalues within the disc $D$ of being either locked or free is central to the structure of the level sets; and, by the counting lemma, this feature is restricted to within the $\operatorname{disc} D$.

\section{5. An Ordering of the Spectrum}

In this subsection we will use counting lemma 1 to introduce a natural ordering for the critical points $\left\{\lambda_{j}^{c}\right\}$. To understand this ordering, fix $\vec{q}, N$ and a disc $D$ by the 
counting lemmas. (Refer to Fig. 2.2). Outside of the disc $D$, the critical points are real; thus, for $|j|>N$,

$$
\begin{gathered}
\lambda_{-N-1}^{c}>\lambda_{-N-2}^{c}>\ldots, \\
\lambda_{N+1}^{c}<\lambda_{N+2}^{c}<\ldots
\end{gathered}
$$

with $\lambda_{j}^{c} \cong j \pi$. The remaining $(2 N+1)$ critical points $\left\{\lambda_{j}^{c}\right\},|j| \leq N$, lie inside the disc $D$. Here a "natural ordering" is not so clear; however, the $2 N+1$ critical points are either real, or they occur in complex conjugate pairs. Thus, there are an odd number $2 M+1$ of real critical points, $M \leq N$. With this in mind, we fix $\vec{q}$, and complete the ordering:

real, inside of $D$

$$
\lambda_{-M}^{c} \leq \lambda_{-M+1}^{c} \leq \ldots \leq \lambda_{0}^{c} \leq \lambda_{1}^{c} \ldots \leq \lambda_{M}^{c}
$$

complex, upper half plane, inside of $D$

$$
\lambda_{j}^{c}, \quad j=M+1, \ldots, N
$$

complex, lower half plane, inside of $D$

$$
\lambda_{-j}^{c}=\bar{\lambda}_{j}^{c} ; \quad j=M+1, \ldots, N .
$$

Let $\vec{\psi}^{ \pm}(x, \lambda)$ denote the Bloch eigenfunctions of $\hat{L}$ at $[\vec{q}, \lambda]$. These eigenfunctions are defined (up to normalization) by the transfer condition across one period:

$$
\vec{\psi}(x+1, \lambda)=\varrho(\lambda) \vec{\psi}(x, \lambda) .
$$

Here $\varrho(\lambda)$ denotes the Floquet multiplier, which is related to the Floquet discriminant by

$$
\varrho(\lambda)=\frac{1}{2}\left[\Delta(\lambda)+\sqrt{\Delta^{2}(\lambda)-4}\right] .
$$

$\varrho$ and $\vec{\psi}$ are well defined functions on the Riemann surface for $\left(\lambda, \sqrt{\Delta^{2}(\lambda)-4}\right)$, and $\vec{\psi}^{ \pm}(x, \lambda)$ denote the values of $\vec{\psi}$ on the two sheets over $\lambda$. At branch points (simple periodic or antiperiodic points), the two sheets touch and $\vec{\psi}^{ \pm}$become linearly independent. (This is compatible with the fact that at a simple eigenvalue, the eigenspace is one dimensional.) At real multiple points, $\vec{\psi}^{ \pm}$remain linearly dependent, while at complex multiple points they may, but need not, become dependent. These two possibilities at the complex multiple points are the key to this nonselfadjoint spectral problem, and are intimately related to the fact that the transfer matrix cannot always be diagonalized at a complex multiple point. (At a complex multiple point, one can only guarantee that it can be placed in Jordan normal form.)

In any case, for fixed $\lambda$, these Bloch eigenfunctions can be represented explicitly in terms of the columns of the fundamental matrix $M(x ; \lambda)=\operatorname{column}\left\{Y^{(1)}(x ; \lambda)\right.$, $\left.Y^{(2)}(x ; \lambda)\right\}$ :

$$
\vec{\psi}^{ \pm}(x ; \lambda)=\alpha^{ \pm}\left\{M_{21}(1 ; \lambda) Y^{(1)}(x ; \lambda)+\left[M_{22}(1 ; \lambda)-\varrho^{ \pm}(\lambda)\right] Y^{(2)}(x ; \lambda)\right\},
$$

where the choice

$$
\alpha^{ \pm}=e^{i \pi / 4} \sqrt{\frac{M_{11}(1, \lambda)-\varrho^{ \pm 1}}{M_{21}(1, \lambda)\left[\Delta^{2}(\lambda)-4\right]}}
$$

guarantees the important symmetry

$$
\psi^{-}(x ; \bar{\lambda})= \pm\left[J \bar{\psi}^{+}(x ; \lambda)\right] .
$$


Remark 1. In order to illustrate these eigenfunctions, it is useful to consider two trivial examples. First, consider the case $\vec{q}=\overrightarrow{0}$. In this case, the multiple points are all real, $\lambda_{j}^{d}=j \pi$, with geometric multiplicity 2 ; the Bloch eigenfunctions for the Lax system are given by

$$
\begin{gathered}
\left(\begin{array}{l}
\psi_{1}^{(+)} \\
\psi_{2}^{(+)}
\end{array}\right)=\exp i\left(\lambda x+2 \lambda^{2} t\right)\left(\begin{array}{l}
1 \\
0
\end{array}\right), \\
\left(\begin{array}{c}
\psi_{1}^{(-)} \\
\psi_{2}^{(-)}
\end{array}\right)=\exp -i\left(\lambda x+2 \lambda^{2} t\right)\left(\begin{array}{l}
0 \\
1
\end{array}\right) .
\end{gathered}
$$

Next, we consider the case of $q(x, t)$ constant, independent of $x$ :

$$
q(x, t)=c \exp \left[-i\left(2 c^{2} t+\gamma\right)\right]
$$

In this case, the Bloch eigenfunctions are given by

$$
\left(\begin{array}{c}
\psi_{1}^{(+)} \\
\psi_{2}^{(+)}
\end{array}\right)=\exp \{ \pm i[\kappa(\lambda)(x+2 \lambda t)]\}\left(\begin{array}{c}
c \exp \left[-i\left(2 c^{2} t+\gamma\right) / 2\right] \\
( \pm \kappa(\lambda)-\lambda) \exp \left[i\left(2 c^{2} t+\gamma\right) / 2\right]
\end{array}\right)
$$

In these formulas, $\kappa(\lambda)$ and $\lambda_{j}$ are given by

$$
\begin{aligned}
\kappa(\lambda) & =\int_{i c}^{\lambda}\left[\frac{\Delta^{\prime}}{\sqrt{\left(\Delta^{2}-4\right)}}\right] d \lambda=\sqrt{\lambda^{2}+c^{2}}, \\
\kappa\left(\lambda_{j}\right) & =j \pi .
\end{aligned}
$$

This example, of $q(x, t)$ constant, independent of $x$, is very useful for illustrating several crucial points. First, the spectrum of the linear operator $\hat{L}$ for coefficients independent of $x$ is easily computed from the Floquet discriminant

$$
\begin{aligned}
\Delta[\lambda ; \vec{q}(\cdot, t ; c, \gamma)] & =2 \cos \kappa(\lambda) \\
& =2 \cos \left[\left(\lambda^{2}+c^{2}\right)^{1 / 2}\right] .
\end{aligned}
$$

This spectrum is depicted in Fig. 2.3.

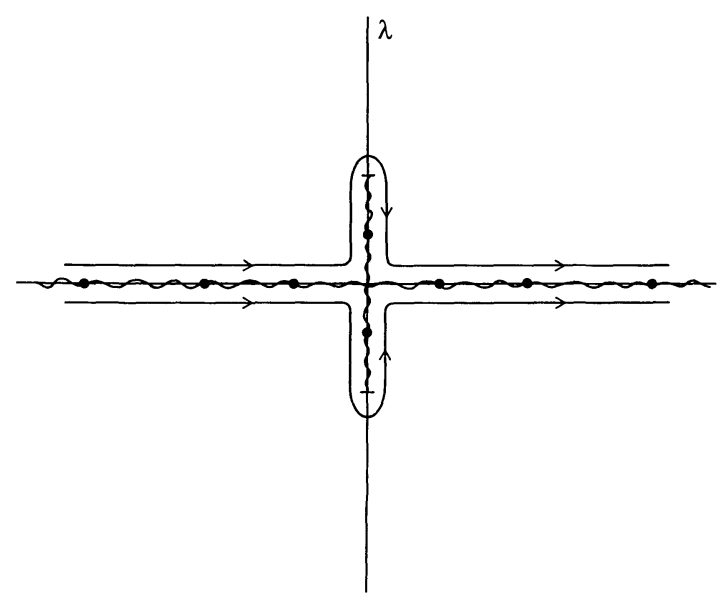

Fig. 2.3. Paths which order the spectrum for the constant potential 
Remark 2. At a solution $q(x, t)$ of NLS, quadratic products of these eigenfunctions can be used to construct solutions of the linearization of NLS about $q(x, t)$. Moreover, these solutions form a "biorthogonal" basis for $\mathscr{F}[24,7,19,2]$. From this basis of linearized solutions, one can infer linearized stability and instabilities of $q(x, t)$. For example, in the case (2.12) when one examines the elements of the basis which are labeled by double points in the spectrum, those associated with real double points are temporally oscillatory, with no growth in time. These solutions of the linearization represent neutrally stable linear modes. On the other hand, those elements associated with complex (in this case, purely imaginary) double points grow exponentially in time $t$ at a rate

$$
\sigma_{j}=4\left|\lambda_{j}\right| \kappa\left(\lambda_{j}\right) .
$$

Such instabilities, associated with complex double points in the spectrum of $\hat{L}$, will play a central role in this paper.

Remark 3. One can ask for a natural ordering of these basis. For self adjoint problems, eigenfunctions are naturally ordered by their oscillations; however, for nonselfadjoint problems, such general orderings do not necessarily exist. Here, due to the counting lemmas, a natural ordering certainly exists for the eigenfunctions associated with spectrum outside of the disc $D$, leaving $2(2 N+1)$ members of the basis to be ordered. For fixed $\vec{q} \in \mathscr{F}$ any prescribed labeling of these $2(2 N+1)$ elements is valid; however, it is intriguing to ask how a prescribed labeling depends upon $\vec{q}$. One possibility, which is really an attempt to extend oscillation theory to this nonselfadjoint setting, is to use the function $\kappa(\lambda)$ to construct an ordering [13,7]. To illustrate this possibility, fix $\vec{q}_{(c, \gamma)}=\vec{q}(\cdot, \cdot ; c, \gamma)$ and view $\kappa\left[\lambda ; \vec{q}_{(c, \gamma)}\right]$ as an Abelian integral on the Riemann surface associated with $\sqrt{\left(\lambda^{2}+c^{2}\right)}$. We fix a contour of integration to make this Abelian integral single valued. For this example, it is sufficient to consider two contour integrals on the complex $\lambda$ plane, one for each sheet on the Riemann surface:

$$
\begin{aligned}
\kappa^{ \pm}\left[\lambda ; \vec{q}_{(c, \gamma)}\right] & =\int_{ \pm i c}^{\lambda}\left[\frac{\Delta^{\prime}}{\sqrt{\left(\Delta^{2}-4\right)}}\right] d \lambda \\
& =\sqrt{\lambda^{2}+c^{2}}
\end{aligned}
$$

where the integration is taken along the two contours depicted in Fig. 2.3. These contour integrals fix an ordering at $\vec{q}_{(c, \lambda)}$ which can be uniquely extended to a small neighborhood of $\vec{q}_{(c, \lambda)}$. This neighborhood is finite. Its size is fixed by the integer $N$ of the counting lemmas as follows: One locates the closest potential $\vec{q}_{*}$ to $\vec{q}_{(c, \lambda)}$ (in $H^{1}$ ) for which there exists a critical point $\lambda_{j}^{c}$ in the disc $D$ such that $\Delta^{\prime \prime}\left(\lambda_{j}^{c}\right)=0$. $\delta$ is then defined as the $H^{1}$ distance from $\vec{q}_{*}$ to $\vec{q}_{(c, \lambda)}$. This distance $\delta$ is positive (not zero) because there are only a finite number $(2 N+1)$ critical points to check. One then chooses the neighborhood to be a ball of radius $r<\delta$. Unfortunately, this ordering cannot be extended unambiguously to a global one valid throughout the $H^{1}$ ball of the counting lemma. It would be very interesting to understand the monodromy associated with this extension procedure, which because of the counting lemma is a finite dimensional problem.

\subsection{The First Variations of $\Delta(\lambda ; \vec{q})$}

Since the Floquet discriminant is entire in both $\vec{q}$ and $\lambda$, it has derivatives which can be calculated by "variation of parameters" (see, for example, [27]): 


\section{Lemma 2.}

$$
\begin{gathered}
\frac{\delta}{\delta \vec{q}} \Delta(\lambda ; q, r)=i \frac{\sqrt{\Delta^{2}-4}}{W\left[\psi^{+}, \psi^{-}\right]}\left[\begin{array}{c}
\psi_{2}^{+}(x ; \lambda) \psi_{2}^{-}(x ; \lambda) \\
\psi_{1}^{+}(x ; \lambda) \psi_{1}^{-}(x ; \lambda)
\end{array}\right] \\
\frac{d \Delta}{d \lambda}=-i \frac{\sqrt{\Delta^{2}-4}}{W\left[\psi^{+}, \psi^{-}\right]} \int_{0}^{1}\left[\psi_{1}^{+} \psi_{2}^{-}+\psi_{2}^{+} \psi_{1}^{-}\right] d x
\end{gathered}
$$

In these formulas, the Bloch eigenfunctions $\psi^{ \pm}$are given explicitly by Eq. (2.10).

\section{Critical Structure of $\mathbf{F}_{j}$}

We fix $\vec{q} \in \mathscr{F}$, as well as a small neighborhood of $\vec{q}, N_{b}(\vec{q})$. In terms of the ordering of Sect. 2.5, we introduce the important sequence of functionals:

$$
\mathbf{F}_{j}: N_{b}(\vec{q}) \rightarrow \mathbf{C} \quad \text { by } \quad \mathbf{F}_{j} \equiv \Delta\left(\lambda_{j}^{c}(\vec{q}) ; \vec{q}\right)
$$

Two points must be emphasized: First, for $|j|>N, \mathbf{F}_{j}$ is real and $\mathbf{F}_{j} \in[-2,+2]$; second, for complex critical points $|j|=M+1, \ldots, N, \mathbf{F}_{j}=\mathbf{F}_{j}^{R}+i \mathbf{F}_{j}^{I}$ is not necessarily real. Thus, it will sometimes be convenient to use an equivalent sequence of real valued constants $\mathbf{G}_{\mathbf{j}}$ :

$$
\mathbf{G}_{j}= \begin{cases}\mathbf{F}_{\mathbf{j}}, & |\mathbf{j}| \leq \mathbf{M}, \\ \frac{1}{2}\left[\mathbf{F}_{\mathbf{j}}+\mathbf{F}_{-\mathbf{j}}\right], & \mathbf{j}=\mathbf{M}+\mathbf{1}, \ldots, \mathbf{N}, \\ \frac{1}{2 i}\left[\mathbf{F}_{\mathbf{j}}-\mathbf{F}_{-\mathbf{j}}\right], & \mathbf{j}=-\mathbf{M}-\mathbf{1}, \ldots,-\mathbf{N}, \\ (-1)^{j} \mathbf{F}_{j}, & |j|>\mathbf{N} .\end{cases}
$$

\subsection{First Variational Condition}

We fix $j$, and, for $\vec{q} \in N_{b}$ throughout which $\Delta^{\prime \prime}\left(\lambda_{j}^{c}\right) \neq 0$, we study critical points of the functional $\mathbf{F}_{\mathbf{j}}$. Formulas 2.14 and 2.13 , together with the fact that $\lambda^{c}(\vec{q})$ is differentiable because it is a simple zero of $\Delta^{\prime}$, immediately yield the following representation of $\operatorname{grad} \mathbf{F}_{j}$ :

\section{Lemma 3.}

$$
\operatorname{grad} \mathbf{F}_{j}(q, r)=i \frac{\sqrt{\Delta^{2}-4}}{W\left[\psi^{+}, \psi^{-}\right]}\left[\begin{array}{l}
\psi_{2}^{+}\left(x ; \lambda_{j}^{c}\right) \psi_{2}^{-}\left(x ; \lambda_{j}^{c}\right) \\
\psi_{1}^{+}\left(x ; \lambda_{j}^{c}\right) \psi_{1}^{-}\left(x ; \lambda_{j}^{c}\right)
\end{array}\right] .
$$

Remark 1. Formula (3.2) for the $\operatorname{grad} \mathbf{F}_{j}$ is actually valid even if $\Delta^{\prime \prime}\left[\lambda_{j}^{c}(\vec{q}) ; \vec{q}\right]=0$, as a limiting calculation shows.

Remark 2. The functions $\vec{q}_{b} \in \mathscr{F}$ at which $\Delta^{\prime \prime}\left[\lambda_{\jmath}^{c}\left(\vec{q}_{b}\right) ; \vec{q}_{b}\right]=0$ are branch points for the functionals $\mathbf{F}_{j}(\vec{q})$. This branching presents challenging obstacles to a global theory (see Sect. 5). When working with the functionals $\mathbf{F}_{j}(\vec{q})$, it is judicious to avoid a branch point $\vec{q}=\vec{q}_{b}$ with the condition $\Delta^{\prime \prime}\left[\lambda_{j}^{c}(\vec{q}) ; \vec{q}\right] \neq 0$. 
For real critical points, the invariant $\mathbf{F}_{j}(\vec{q})$ is a real valued functional on $\mathscr{F}$; however, for complex critical points, this invariant is complex valued and we view the functional as two real valued ones:

$$
\mathbf{F}_{j}(\vec{q})=\mathbf{F}_{j}^{R}(\vec{q})+i \mathbf{F}_{j}^{I}(\vec{q}) .
$$

These two invariants are functionally independent, as the following lemma states.

Lemma 4. For a complex critical point $\lambda_{j}^{c}(\vec{q})$, we assume that $\left.\operatorname{grad} \mathbf{F}_{j}\right|_{q} \neq 0$, and that $q$ and $q_{x}$ are linearly independent as functions of $x$. Then $\mathbf{F}_{j}^{R}$ and $\mathbf{F}_{j}^{I}$ are functionally independent.

Proof. It's sufficient to prove that $\mathbf{F}_{\mathbf{j}}$ and $\overline{\mathbf{F}}_{\mathbf{j}}$ are functionally independent. The complex critical points have been ordered so that $\bar{\lambda}_{j}^{c}=\lambda_{-j}^{c}$. This ordering, together with representation (3.2) for the first variation of $\mathbf{F}_{j}$, shows that we need only to prove that $\left(\psi^{+} \psi^{-}\right)\left(\lambda_{j}^{c}\right)$ and $\left(\psi^{+} \psi^{-}\right)\left(\bar{\lambda}_{j}^{c}\right)$ are linearly independent. The assumption that they are linearly dependent, together with the squared eigenfunction equation, implies that either $q$ and $q_{x}$ are linearly dependent or $\bar{\lambda}_{j}^{c}=\lambda_{j}^{c}$ - thus establishing the lemma.

Next, for fixed $j$, we consider critical potentials $\vec{q}_{*}$ for which

$$
\left.\operatorname{grad} \mathbf{F}_{\mathbf{j}}\right|_{q_{*}}=0 \text {. }
$$

The following lemma is seen to be valid, mostly by inspection of formula (3.2):

Lemma 5. Except for the trivial case $\vec{q}=\overrightarrow{0}$,

$$
\begin{aligned}
\frac{\delta \mathbf{F}_{j}}{\delta q}=0 & \Leftrightarrow \\
\frac{\delta \mathbf{F}_{j}}{\delta \vec{q}}=0 & \Leftrightarrow M\left(1 ; \lambda_{j}^{c}, \vec{q}_{*}\right)= \pm I . \\
\left.\operatorname{grad} \mathbf{F}_{j}\right|_{q_{*}}=0 & \Rightarrow \Delta^{\prime}\left(\lambda_{j}^{c}\left(\vec{q}_{*}\right) ; \vec{q}_{*}\right)=0, \\
& \Rightarrow\left|\mathbf{F}_{j}\left(\vec{q}_{*}\right)\right|=2, \\
& \Rightarrow \lambda_{j}^{c}\left(\vec{q}_{*}\right) \text { is a multiple point. }
\end{aligned}
$$

Remark. The reverse implications for statement 2 of this lemma are not valid. There exists $\vec{q} \in \mathscr{F}$ with a (complex) double point $\lambda_{j}^{d}(\vec{q})$ at which $\mathbf{F}_{\jmath}=2$ and $\Delta^{\prime}=0$, and yet $\operatorname{grad} \mathbf{F}_{j} \neq 0$. Indeed, such potentials, which can be constructed with Bäcklund transformations (see Sect. 4.2), are at the heart of a hyperbolic structure which is central to our theory.

Remark. In the exceptional $\vec{q}=\overrightarrow{0}$ case, $\operatorname{grad} \Delta$ vanishes identically in $\lambda$, as is apparent from (3.2).

Remark. The facts that

$$
\begin{aligned}
\frac{\delta}{\delta q} \mathbf{F}_{j}^{R} & =0 \Leftrightarrow \frac{\delta}{\delta q} \mathbf{F}_{j}^{I}=0 ; \\
\frac{\delta}{\delta q} \mathbf{F}_{j}^{R} & =0 \Rightarrow \mathbf{F}_{j}^{I}=0
\end{aligned}
$$

are immediate consequences of Lemma 5 . Fact 1 states that $\mathbf{F}_{j}^{R}$ and $\mathbf{F}_{j}^{I}$ share the same critical manifold. Fact 2 states that critical functions of $\mathbf{F}_{j}^{R}$ must lie on the level set $\mathbf{F}_{j}^{I}=0$. These two facts will be important for a Morse theory for NLS level sets, as discussed in Sect. 5. 


\subsection{Critical Tori}

Assume that neighborhood $N_{b}$ contains an " $N$-phase" potential $\vec{q}_{N}$ and is small enough that, for typical $\vec{q} \in N_{b}$, the isospectral level sets consist of (infinite) dimensional tori; the critical points $\vec{q}_{*}$ lie on "singular" tori, with one or more of the circles pinched off. (One circle is pinched off for each critical $\mathbf{F}_{\mathbf{j}}$ associated to a real critical point, while several circles may be pinched for each critical $\mathbf{F}_{\mathbf{j}}$ associated with a complex critical point.) These singular tori themselves are lower dimensional tori, with or without "whiskers." The existence of these critical tori is guaranteed by inverse spectral theory which can be used to construct some of them in terms of finite genus theta functions. (The remaining critical tori could be obtained through limits to infinite genus theta functions, and the whiskered tori can be constructed by Bäcklund transformations of the singular tori as discussed in Sect. 4.2.) It would be interesting to construct these critical tori directly from variational problem (3.3), without invoking inverse spectral theory except in a context which is natural to that variational problem. However, we have not done so. Rather, to construct critical tori, we have freely used results from the general theory of the inverse spectral transform.

\subsection{The Hessian of $F_{j}$}

For fixed $j$ and $\vec{q} \in N_{b}$ in which $\Delta^{\prime \prime}\left(\lambda_{j}^{c}\right) \neq 0$, the critical points of $\mathbf{F}_{\mathbf{j}}$ consist in a subset $S_{j} \subset N_{b}$ such that

$$
\left.\operatorname{grad} \mathbf{F}_{j}\right|_{q_{*}}=0, \quad \vec{q}_{*} \in S_{j} .
$$

$\mathbf{F}_{\mathbf{j}}$ has critical values of \pm 2 on $S_{\jmath}$. Here we fix $\vec{q}_{*} \in S_{j}$, and consider the Hessian of $\mathbf{F}_{\mathbf{j}}$ at $\vec{q}_{*}$.

This Hessian can be expressed in terms of quadratic products of eigenfunctions (2.10) at the double point $\lambda_{j}^{d}$, with a different normalization which is valid in a neighborhood of $\lambda_{j}^{d}$, provided $\Delta^{\prime \prime}\left(\lambda_{j}^{d}\right) \neq 0$ :

$$
\alpha^{+}=\alpha^{-}=\frac{i}{2\left(\lambda-\lambda_{j}^{d}\right) \sqrt{b \Delta^{\prime \prime}\left(\lambda_{j}^{d}\right)}} .
$$

Here the coefficient $b$ (assumed $\neq 0$ ) is given by

$$
b=\int_{0}^{1} M_{12} M_{22} d x .
$$

It will also be useful to define two additional coefficients

$$
\begin{aligned}
& a=\int_{0}^{1} M_{11} M_{21} d x, \\
& c=\int_{0}^{1}\left[M_{11} M_{22}+M_{12} M_{21}\right] d x .
\end{aligned}
$$


The following relationship between these coefficients can be established:

$$
\Delta^{\prime \prime}\left(\lambda_{j}^{d}\right)=\Delta\left(\lambda_{j}^{d}\right)\left[4 a b-c^{2}\right] .
$$

In terms of these coefficients, one computes (see [20]) two squared eigenfunctions:

$$
\begin{aligned}
\chi^{(+)} & =\left[\psi^{(+)}\right]^{2}+\left[\psi^{(-)}\right]^{2} \\
& =\left[\Delta^{\prime \prime}\right]^{-1 / 2}\left\{-\frac{\Delta^{\prime \prime}\left(\lambda_{j}^{d}\right)}{b \Delta\left(\lambda_{j}^{d}\right)}\left(Y^{(2)}\right)^{2}+2\left[a\left(Y^{(2)}\right)^{2}-c\left(Y^{(1)} Y^{(2)}\right)+b\left(Y^{(1)}\right)^{2}\right]\right\} \\
\chi^{(-)} & =\left[\psi^{(+)}\right]^{2}-\left[\psi^{(-)}\right]^{2} \\
& =\sqrt{2 i}\left[M_{11}(1)\right]^{3 / 2}\left[\frac{c}{2 b}\left(Y^{(2)}\right)^{2}-Y^{(1)} Y^{(2)}\right] .
\end{aligned}
$$

If one decomposes $\chi^{( \pm)}$into $\mathscr{F} \oplus i \mathscr{F}$.

$$
\chi^{ \pm}=\chi_{f}^{ \pm}+\chi_{i f}^{ \pm}
$$

then $\chi_{f}^{ \pm}$and $i \chi_{i f}^{ \pm} \in \mathscr{F}$. Finally, we define the projections

$$
\begin{aligned}
& \delta \Xi_{1}=\left\langle-i K \chi_{i f}^{+}, \delta \vec{q}\right\rangle, \\
& \delta \Xi_{2}=\left\langle K \chi_{f}^{-}, \delta \vec{q}\right\rangle, \\
& \delta \Xi_{3}=\left\langle K \chi_{f}^{+}, \delta \vec{q}\right\rangle, \\
& \delta \Xi_{4}=\left\langle-i K \chi_{i f}^{-}, \delta \vec{q}\right\rangle .
\end{aligned}
$$

The symmetries satisfied by the $\chi$ 's, together with the fact that $\delta \vec{q} \in \mathscr{F}$, show that these projections $\delta \Xi$ are real. In order to understand why these projections are natural, recall that "squared eigenfunctions" generate a biorthogonal basis. When expanding $\delta \vec{q}$ in terms of the linearized basis, the expansion coefficients are computed by projections onto members of its adjoint basis.

In addition to the reality of the projections, a second important property is the dimension of the span of $\left\{\chi_{f}^{+}, \chi_{f}^{-}, i \chi_{i f}^{+}, i \chi_{i f}^{-}\right\}$. In general, at a complex double point of geometric multiplicity 2 , these four functions are linearly independent and the dimension is four. On the other hand, at a real double point, the span is two dimensional, and one has the following dependencies:

$\lambda_{\jmath}^{d}$ real, $M_{11}=-1\left(\Rightarrow \Delta^{\prime \prime}\left(\lambda_{j}^{d}\right)>0\right)$ :

$$
\chi_{f}^{+}=\Gamma^{-1} \chi_{f}^{-}, \quad \chi_{i f}^{+}=\Gamma \chi_{i f}^{-} ;
$$

$\lambda_{j}^{d}$ real, $M_{11}=1\left(\Rightarrow \Delta^{\prime \prime}\left(\lambda_{j}^{d}\right)<0\right)$ :

$$
\chi_{f}^{+}=\Gamma \chi_{f}^{-}, \quad \chi_{\imath f}^{+}=\Gamma^{-1} \chi_{i f}^{-},
$$

where $\Gamma$ is defined by

$$
\Gamma \equiv \sqrt{\frac{\left|\Delta^{\prime \prime}\right|}{2 c^{2}}} .
$$

A calculation (see [20]) then yields 
Theorem 2. Let $\vec{q}$ denote a critical point of $\mathbf{F}_{\mathbf{j}}$. The Hessian of $\mathbf{F}_{\mathbf{j}}$ at $\vec{q}$ is given by 1. $\lambda_{j}^{d}$ real:

$$
\begin{array}{ll}
\delta^{2} \mathbf{F}_{j}=\delta^{2} \mathbf{F}_{j}^{R}=\frac{8|a|^{2}}{\Delta^{\prime \prime}\left(\lambda_{j}^{d}\right)}\left[\left(\delta \Xi_{1}\right)^{2}+\left(\delta \Xi_{2}\right)^{2}\right], & \Delta^{\prime \prime}>0 ; \\
\delta^{2} \mathbf{F}_{j}=\delta^{2} \mathbf{F}_{j}^{R}=\frac{8|a|^{2}}{\Delta^{\prime \prime}\left(\lambda_{j}^{d}\right)}\left[\left(\delta \Xi_{3}\right)^{2}+\left(\delta \Xi_{4}\right)^{2}\right], & \Delta^{\prime \prime}<0 .
\end{array}
$$

2. $\lambda_{j}^{d}$ complex:

$$
\begin{aligned}
\delta^{2} \mathbf{F}_{j}^{R} & =\left[\left(\delta \Xi_{1}\right)^{2}+\left(\delta \Xi_{2}\right)^{2}\right]-\left[\left(\delta \Xi_{3}\right)^{2}+\left(\delta \Xi_{4}\right)^{2}\right] \\
\delta^{2} \mathbf{F}_{j}^{I} & =2\left[\left(\delta \Xi_{2}\right)\left(\delta \Xi_{4}\right)-\left(\delta \Xi_{1}\right)\left(\delta \Xi_{3}\right)\right] .
\end{aligned}
$$

For applications, even functions of $x$ are particularly important. When both $\vec{q}(x)$ and $\delta \vec{q}(x)$ are even functions of $x$, the projections $\delta \Xi$ become dependent and the following corollary results.

Corollary 1. Let $\vec{q}(x)$ and $\delta \vec{q}(x)$ be even functions of $x$, and consider a purely imaginary complex double point $\lambda_{j}^{d}$. One has

$$
\begin{aligned}
\delta^{2} \mathbf{F}_{\mathbf{j}} & =\delta^{2} \mathbf{F}_{\mathbf{j}}^{\mathbf{R}} \\
& =\left\{\begin{array}{lll}
\frac{4 a \bar{a}}{c^{2}}\left(\delta \Xi_{2}^{2}-\delta \Xi_{3}^{2}\right), & \Delta\left(\lambda_{j}^{d}\right)=-2, & \frac{\Delta^{\prime \prime}\left(\lambda_{j}^{d}\right)}{\Delta\left(\lambda_{j}^{d}\right)}>0 ; \\
\frac{4 a \bar{a} \Delta\left(\lambda_{j}^{d}\right)}{\Delta^{\prime \prime}\left(\lambda_{j}^{d}\right)}\left(\delta \Xi_{2}^{2}-\delta \Xi_{3}^{2}\right), & \Delta\left(\lambda_{j}^{d}\right)=2, & \frac{\Delta^{\prime \prime}\left(\lambda_{j}^{d}\right)}{\Delta\left(\lambda_{j}^{d}\right)}>0 ; \\
-\frac{4 a \bar{a} \Delta\left(\lambda_{j}^{d}\right)}{\Delta^{\prime \prime}\left(\lambda_{j}^{d}\right)}\left(\delta \Xi_{3}^{2}+\delta \Xi_{4}^{2}\right), & \Delta\left(\lambda_{j}^{d}\right)=-2, & \frac{\Delta^{\prime \prime}\left(\lambda_{j}^{d}\right)}{\Delta\left(\lambda_{j}^{d}\right)}<0 ; \\
\frac{-4 a \bar{a}}{c^{2}}\left(\delta \Xi_{3}^{2}+\delta \Xi_{4}^{2}\right), & \frac{\Delta^{\prime \prime}\left(\lambda_{j}^{d}\right)}{\Delta\left(\lambda_{j}^{d}\right)}<0 .
\end{array}\right.
\end{aligned}
$$

Remark. For $\vec{q}$ independent of $x$, one has $\Delta^{\prime \prime}\left(\lambda_{j}^{d}\right) / \Delta\left(\lambda_{j}^{d}\right)>0$ when $\lambda=-\bar{\lambda}$. We suspect, but have not been able to establish, this condition for even functions $\vec{q}$, with $\lambda=-\bar{\lambda}$.

Remark. Examples indicate that the Hessians in (3.7) and (3.8) actually have limits at the branch points, $\vec{q} \rightarrow \vec{q}_{b}$. We will not make use of this regularity, however,

\subsection{The Hessian and Passage Through Spectral Singularities}

The Hessian of $\mathbf{F}_{\mathbf{j}}$, as summarized in Theorem 2, contains considerable information about the structure of the spectrum of $\hat{L}$ even though it involves only local information in a small neighborhood of $\vec{q}_{*}$. In particular, it enables us to describe spectral configurations near singular ones. The passage through these spectral singularities occurs in the numerical experiments on perturbed NLS equations and is central to the chaotic behavior observed in those experiments. We conclude this section with a brief discussion of the Hessian and these spectral configurations. 
First, consider the information about the spectrum of $\hat{L}$ which is contained in this Hessian. At a real critical point $\lambda_{c}^{j}, \mathbf{F}_{j}$ has a maximum of +2 (or a minimum value of -2 ) at a critical potential $\vec{q}_{*}$. In a neighborhood of $\vec{q}_{*}$, the value of $\mathbf{F}_{j}$ can only decrease (increase). But $\mathbf{F}_{j}$ can be interpreted as the height of the Floquet discriminant $\Delta(\lambda)$ over the critical point $\lambda_{c}^{j}$. This fact, together with the saddle structure of the real part of the analytic function $\Delta(\lambda)$, shows that, in a neighborhood of the critical point $\lambda_{c}^{j}$, no gaps on the real axis can appear in the spectrum of $\hat{L}$; that at the critical point $\lambda_{c}^{j}$, a spine of spectrum bifurcates off the real axis into the complex $\lambda$ plane; and that the length of the $j^{\text {th }}$ spine vanishes when $\mathbf{F}_{j}$ is critical as a functional of $\vec{q}$. Typical "spine-like" spectral configurations are depicted in Fig. 3.1.

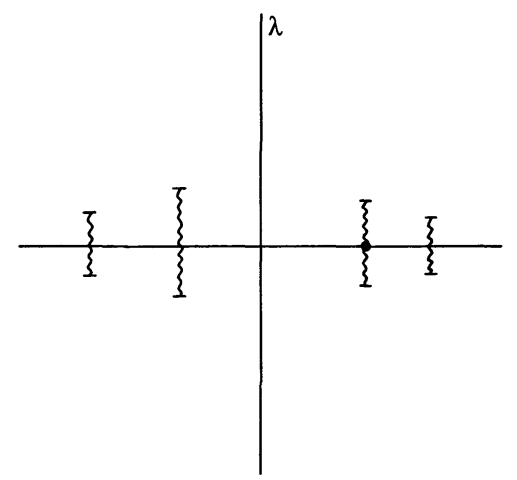

Fig. 3.1. Typical "spine-like" spectral configuration

Much more interesting behavior is associated to complex critical points. At a complex critical point $\lambda_{c}^{j}$, Theorem 2 shows that $\mathbf{F}_{j}$ has a saddle structure as a functional of $\vec{q}$. While $\mathbf{F}_{j}$ still has critical value $+2(-2)$ at a critical potential $\vec{q}_{*}$, its saddle structure in a neighborhood of $\vec{q}$ has striking consequences which are easiest to demonstrate in the case of a purely imaginary critical point $\lambda_{c}^{j}$ under the constraint of even potentials $\vec{q}(x)$. Under these constraints, $\mathbf{F}_{j}$ is real valued in a neighborhood of $\vec{q}_{*}$, and can still be interpreted as the height of the Floquet discriminant over the critical point $\lambda_{c}^{j}$. Near $\vec{q}_{*},\left|\mathbf{F}_{\jmath}\right|$ can increase above 2 and a gap can develop in the purely imaginary band of spectrum; for other $\vec{q}$ near $\vec{q}_{*},\left|\mathbf{F}_{j}\right|$ can drop below 2 and a spine in the spectrum can bifurcate at $\lambda_{c}^{j}$ into the first and second quadrants of the complex $\lambda$ plane. That is, the spectrum configuration can develop a "cross-like" structure. (See Fig. 5.5.) Furthermore, when the constraints are dropped, still more complicated spectral configurations emerge as consequences of the saddle structure of the Hessian [27]. Such behavior of the functionals $\left\{\mathbf{F}_{j}\right\}$ should help in understanding the "tree structure" in the spectrum of the nonselfadjoint operator $\hat{L}$.

In addition to spectral information, one can also extract information about the NLS level sets in function space $\mathscr{F}$ from this Hessian. Consider a $\vec{q} \in \mathscr{F}$ for which the isospectral set $\mathscr{Q}(\vec{q})$ is an (infinite) product of circles. At issue here is how these tori change with $\vec{q}$, that is with the (values of) the spectrum, and how they stratify (or fill out) the phase space $\mathscr{F}$. Clearly, such a stratification by tori will be organized by neighborhoods of those "critical tori" for which one or more of the circles in the infinite product has "pinched off." 
Our idea is to use the functionals $\mathbf{F}_{j}(\vec{q})$ to describe this stratification. The vanishing of the first variation should determine the critical tori, and the Hessian should determine the "saddle" or "center" of these critical tori. Before discussing the possibility of such a Morse theory of NLS level sets in more detail, we first use Bäcklund transformations to construct the critical level sets; more precisely, to construct the stable and unstable manifolds of the critical tori. The indices of the Hessian can be used to count the dimension of these stable and unstable manifolds. These unstable manifolds are called "unstable whiskers;" the stable manifolds are called "stable whiskers;" and the complete structures are called "whiskered tori" [1]. This Bäcklund construction is the topic of the next Sect. 4.

\section{Global Representations of Whiskered Tori}

In dynamical systems theory, one is often primarily interested in temporal instabilities under a particular time flow; here, under the NLS flow generated by the NLS Hamiltonian. The saddle structure unveiled by the above expansion does not guarantee this NLS instability; rather, it shows that certain Hamiltonian flows generated by $\mathbf{F}_{j}$ possess instabilities. Comparison of the Hamiltonian vector fields $J \operatorname{grad} H$ and $J \operatorname{grad} \mathbf{F}$ could assess the temporal instability of NLS; however, we have not done this comparison. Rather, we address the NLS flow directly.

\subsection{Linearized Instabilities}

Fix a solution $\vec{q}(x, t)$ of the NLS equation which is periodic in $x$ and quasiperiodic in $t$; more precisely, fix a $\vec{q}$ on one of the invariant tori. Linearizing NLS about $\vec{q}$ yields a linearized equation. As mentioned in Sect. 2.5, quadratic products of solutions of the Zakharov-Shabat linear system,

$$
\begin{gathered}
{\left[-i \sigma_{3} \frac{d}{d x}-\left(\begin{array}{cc}
0 & q \\
-\bar{q} & 0
\end{array}\right)\right] \vec{\psi}=\lambda \vec{\psi}} \\
{\left[-i \sigma_{3} \frac{d}{d t}+q \bar{q}+\left(\begin{array}{cc}
0 & -2 \lambda q+i q_{x} \\
2 \lambda \bar{q}+i \bar{q}_{x} & 0
\end{array}\right)\right] \vec{\psi}=2 \lambda^{2} \vec{\psi}}
\end{gathered}
$$

generate a basis of solutions of the linearization. With this basis one can assess the linear stability properties of the solution $\vec{q}$. First, (in the absence of higher order multiple points), the basis splits into two parts, one labeled by simple eigenvalues and one labeled by double points. There is no exponential growth associated with that part of the basis associated to the simple eigenvalues, nor to that part associated to real double points. The only possible exponential instabilities are labeled by complex multiple points. By the counting lemma, these are at most finite in number and they reside in the disc $D$. Typically, for each complex double point there is one exponentially growing and one exponentially decaying linearized solution. These instabilities are associated with the saddle structure described above. However, that saddle structure is associated with the topological properties of the critical level set while temporal instabilities are associated with one particular flow, the NLS flow. It can happen that this particular flow does not "pick up" the unstable direction, and thus is accidentally stable. In the case of the sine-Gordon equation, examples exist [10] of complex double points which are indeed associated to instabilities and others which 
are associated to stable behavior for one particular flow. At present, each complex double point must be investigated individually for instability with respect to a given flow. Those linearized solutions which do behave exponentially generate a basis for the (finite dimensional) tangent spaces of the stable and unstable manifolds (for the NLS flow) of the critical torus.

\subsection{Bäcklund Transformations}

Using Bäcklund transformations one can exponentiate these linearized solutions to obtain global solutions of the NLS equation. Fix a periodic solution $\vec{q}(x, t)$ of NLS which is quasiperiodic in $t$, for which the linear operator $\hat{L}$ has a complex double point $\nu$ of geometric multiplicity 2 which is associated with an NLS instability. We denote two linearly independent solutions of the Zakharov-Shabat linear system (4.1) at $\lambda=\nu$ by $\left(\vec{\phi}^{+}, \vec{\phi}^{-}\right)$. Thus, a general solution of the linear system at $(\vec{q}, \nu)$ is given by

$$
\vec{\phi}\left(x, t ; \nu ; c_{+}, c_{-}\right)=c_{+} \vec{\phi}^{+}+c_{-} \vec{\phi}^{-} .
$$

We use $\vec{\phi}$ to define a transformation matrix [30] $G$ by

$$
G=G(\lambda ; \nu ; \vec{\phi}) \equiv N\left(\begin{array}{cc}
\lambda-\nu & 0 \\
0 & \lambda-\bar{\nu}
\end{array}\right) N^{-1}
$$

where

$$
N \equiv\left[\begin{array}{cc}
\phi_{1} & -\bar{\phi}_{2} \\
\phi_{2} & \bar{\phi}_{1}
\end{array}\right]
$$

Then we define $Q$ and $\vec{\Psi}$ by

$$
Q(x, t) \equiv q(x, t)+2(\nu-\bar{\nu}) \frac{\phi_{1} \bar{\phi}_{2}}{\phi_{1} \bar{\phi}_{1}+\phi_{2} \bar{\phi}_{2}}
$$

and

$$
\vec{\Psi}(x, t ; \lambda) \equiv G(\lambda ; \nu ; \vec{\phi}) \vec{\psi}(x, t ; \lambda)
$$

where $\vec{\psi}$ solves the linear system $(4.1)$ at $(\vec{q}, \nu)$. Formulas $(4.5)$ and $(4.6)$ are the Bäcklund transformations for the potential and eigenfunctions, respectively. We have the following

Theorem 3. Let $q(x, t)$ denote a periodic solution of NLS, which is linearly unstable with an exponential instability associated to a complex double point $\nu$ in $\sigma(\hat{L}(\vec{q}))$. Let the complex double point $\nu$ have geometric multiplicity 2 , with eigenbasis $\left(\vec{\phi}^{+}, \vec{\phi}^{-}\right)$ for linear system (4.1), and define $Q(x, t)$ and $\vec{\Psi}(x, t ; \lambda)$ by $(4.5)$ and (4.6) Then

(i) $Q(x, t)$ is an solution of NLS, with spatial period 1 ;

(ii) $\sigma(\hat{L}(\vec{Q}))=\sigma(\hat{L}(\vec{q}))$;

(iii) $Q(x, t)$ is homoclinic to $q(x, t)$ in the sense that $Q(x, t) \rightarrow q_{\theta_{ \pm}}(x, t)$, exponentially as $\exp \left(-\sigma_{\nu}|t|\right)$ as $t \rightarrow \pm \infty$ Here $q_{\theta_{ \pm}}$is a "torus translate" of $q, \sigma_{\nu}$ is the nonvanishing growth rate associated to the complex double point $\nu$ For finite dimensional tori explicit formulas exist for this growth rate and for the translation parameters $\theta_{ \pm}$. (iv) $\vec{\Psi}(x, t ; \lambda)$ solves the linear system $(4.1)$ at $(\vec{Q}, \lambda)$. 
This theorem is quite general, constructing homoclinic solutions from a wide class of starting solutions $\vec{q}(x, t)$. Its proof is one of direct verification, following the sineGordon model [8] with the formalism of [30]. Periodicity in $x$ is achieved by choosing the transformation parameter $\lambda=\nu$ to be a double point.

In references [26] and [27], several qualitative features of these homoclinic orbits are emphasized: (i) $Q(x, t)$ is homoclinic to a torus which itself possesses rather complicated spatial and temporal structure, and is not just a fixed point; (ii) nevertheless, the homoclinic orbit typically has still more complicated spatial structure than its "target torus." (iii) When there are several complex double points, each with nonvanishing growth rate, one can iterate the Bäcklund transformations to generate more complicated homoclinic manifolds. (iv) The number of complex double points with nonvanishing growth rates counts the dimension of the unstable manifold of the critical torus in that two unstable directions are coordinatized by the complex ratio $c_{+} / c_{-}$. Under even symmetry only one real dimension satisfies the constraint of evenness, as will be clearly illustrated in the following example. (v) These Bäcklund formulas provide coordinates for the stable and unstable manifolds of the critical tori; thus, they provide explicit representations of the critical level sets which consist in "whiskered tori."

\subsection{An Example: The Spatially Uniform Plane Wave}

As a concrete example, we return to the spatially uniform plane wave discussed in Remark 1 of Sect. 2.5. From the eigenfunctions given in that remark, one can construct the fundamental matrix

$$
M(x ; \lambda ; C)=\left[\begin{array}{cc}
\cos \kappa x+i \frac{\lambda}{\kappa} \sin \kappa x & i \frac{C}{\kappa} \sin \kappa x \\
i \frac{\bar{C}}{\kappa} \sin \kappa x & \cos \kappa x-i \frac{\lambda}{\kappa} \sin \kappa x
\end{array}\right]
$$

from which the Floquet discriminant can be computed:

$$
\Delta(\lambda ; C)=2 \cos \kappa
$$

From $\Delta$, spectral quantities can be computed:

Simple Periodic Points:

$$
\lambda^{ \pm}= \pm i c
$$

Double Points:

$$
\begin{gathered}
\kappa\left(\lambda_{j}^{d}\right)=j \pi, \quad j \in \mathbf{Z}, \quad j \neq 0 ; \\
\lambda_{j}^{c}=\lambda_{j}^{d}, \quad j \in \mathbf{Z}, \quad j \neq 0 ; \\
\lambda_{0}^{c}=0 .
\end{gathered}
$$

For this spectral data, there are $2 N$ purely imaginary double points,

$$
\left(\lambda_{\jmath}^{d}\right)^{2}=\pi^{2} j^{2}-c^{2}, \quad j=1,2, \ldots, N
$$

where

$$
\left[\pi^{2} N^{2}-c^{2}\right]<0<\left[\pi^{2}(N+1)^{2}-c^{2}\right] .
$$

The linearized basis as mentioned in Sect. 2.5 shows that these complex double points are indeed associated with instabilities, with temporal growth rates given by

$$
\sigma_{\jmath}=4\left|\lambda_{j}^{d}\right| \kappa\left(\lambda_{j}^{d}\right), \quad j=1,2, \ldots, N .
$$


From this spectral data, the homoclinic orbits can be explicitly computed. A single Bäcklund transformation at one purely imaginary double point yields $Q=$ $Q_{H}\left(x, t ; c, \gamma ; k=\pi, c_{+} / c_{-}\right)$:

$$
\begin{aligned}
Q_{H}= & {\left[\frac{\cos 2 p-\sin p \operatorname{sech} \tau \cos (2 k x+\phi)-i \sin 2 p \tanh \tau}{1+\sin p \operatorname{sech} \tau \cos (2 k x+\phi)}\right] } \\
& \times c e^{-i\left(2 c^{2} t+\gamma\right)} \rightarrow e^{\mp 2 i p} c e^{-i\left(2 c^{2} t+\gamma\right)} \text { as } \varrho \rightarrow \mp \infty,
\end{aligned}
$$

where $c_{+} / c_{-} \equiv \exp (\varrho+i \beta)$ and $p$ is defined by $k+\nu=c \exp (i p), \tau \equiv \sigma t-\varrho$, and $\phi \equiv p-(\beta+\pi / 2)$.

Several points about this homoclinic orbit need to be made.

(i) The orbit depends only upon the ratio $c_{+} / c_{-}$, and not upon $c_{+}$and $c_{-}$ individually.

(ii) $Q_{H}$ is homoclinic to the plane wave orbit; however, a phase shift of $-4 p$ occurs when one compares the asymptotic behavior of the orbit as $t \rightarrow-\infty$ with its behavior as $t \rightarrow+\infty$.

(iii) For small $p$, the formula for $Q_{H}$ becomes more transparent:

$$
Q_{H} \cong[(\cos 2 p-i \sin 2 p \tanh \tau)-2 \sin p \operatorname{sech} \tau \cos (2 k x+\phi)] c e^{-i\left(2 c^{2} t+\gamma\right)} .
$$

(iv) The complex transformation parameter $c_{+} / c_{-}=\exp (\varrho+i \beta)$ can be thought of as $S \times R$. In the formula an evenness constraint in $x$ can be enforced by restricting the phase $\phi$ to one of two values -

$$
\phi=0, \pi . \quad \text { (evenness) }
$$

In this manner, evenness reduces the formula for $Q_{H}$ from $S \times R$ to two copies of $R$, and even symmetry disconnects the level set. Each component constitutes one whisker. While the target $q$ is independent of $x$, each of these whiskers has $x$ dependence through the $\cos (2 k x)$. One whisker has exactly this dependence and can be interpreted as a spatial excitation located near $x=0$ - while the second whisker has the dependence $\cos (2 k(x-\pi / 2 k))$, which we interpret as spatial structure located near $x=1 / 2$. In this example, the disconnected nature of the level set is clearly related to distinct spatial structures on the individual whiskers.

In this example the target is always the plane wave; hence, it is always a circle of dimension one, and in this example we are really constructing only whiskered circles. On the other hand, in this example the dimension of the whiskers need not be one, but is determined by the number of purely imaginary double points which in turn is controlled by the amplitude $c$ of the plane wave target and by the spatial period. (The dimension of the whiskers increases linearly with the spatial period.) When there are several complex double points, Bäcklund transformations must be iterated to produce complete representations. While these iterated formulas are quite complicated, their parameterizations admit rather direct qualitative interpretations [31].

Thus, Bäcklund transformations give global representations of the critical level sets. In the next section we discuss neighborhoods of these critical level sets.

\section{Toward a Morse Description of the Isospectral Stratification}

In this section we introduce a Morse theoretic description of the NLS level sets [9]. A goal of such a Morse theoretic study of the integrable NLS system would be to 
understand how the tori and singular tori, which constitute the invariant isospectral sets of $\hat{L}$, stratify the phase space $\mathscr{F}$. This phase space is too large. In order to make use of the control provided by the counting lemmas, we restrict to a smaller invariant subset of $\mathscr{F}$.

First, we use the invariants of $H_{0}$ (the $L^{2}$ norm) and $H_{2}$ (the energy) in order to define a subset $\mathscr{B}^{(h)} \subset \mathscr{F}$ : For fixed positive numbers $h_{0}$ and $h_{2}$,

$$
\mathscr{B}^{(h)} \equiv\left\{\vec{q} \in \mathscr{F}\left|H_{0} \leq h_{0},\right| H_{2} \mid \leq h_{2}\right\},
$$

Sobolev arguments show that the set $\mathscr{B}^{(h)}$ is a closed invariant set which is bounded in $H^{1}$ and therefore compact in $L^{2}$. For $\vec{q} \in \mathscr{B}^{(h)}$, the "counting lemmas" identify an integer $N=N^{(h)}$ and a disc $D=D^{(h)}$ in the complex plane.

For $\vec{q} \in \mathscr{B}^{(h)}$, all critical points $\left\{\lambda_{j}^{c}\right\}_{|j|>N}$ are real and can be ordered without ambiguity $\forall \vec{q} \in \mathscr{B}^{(h)}$ by the counting lemma. On the other hand, for the $2 N+1$ critical points inside the disc $D$, a single valued ordering throughout $\mathscr{B}^{(h)}$ is impossible because of collisions of critical points which occur when $\Delta^{\prime \prime}\left[\lambda_{j}^{c}(\vec{q}) ; \vec{q}\right]=0$. At best, any fixed local ordering, at a fixed $\vec{q} \in \mathscr{B}^{(h)}$, extends throughout $\mathscr{B}^{(h)}$ to a multiple valued ordering involving permutations of the colliding critical points. Following [2], for any fixed $j,|j| \leq N, \lambda_{j}^{c}(\vec{q})$ is a multiple valued analytic function whose values lie in the set $\left\{\lambda_{k}^{c}(\vec{q}) \forall k,|k| \leq N\right\}$. The "branch points" are those functions $\vec{q}_{b} \in \mathscr{B}^{(h)}$ on a variety in $\mathscr{B}^{(h)}$ defined by

$$
\Delta^{\prime}\left[\lambda_{j}^{c}\left(\vec{q}_{b}\right) ; \vec{q}_{b}\right]=\Delta^{\prime \prime}\left[\lambda_{j}^{c}\left(\vec{q}_{b}\right) ; \vec{q}_{b}\right]=0,
$$

for some $|j| \leq N$.

In terms of this ordering, we consider the sequence of functionals:

$$
\mathbf{F}_{j}: \mathscr{B}^{(h)} \rightarrow \mathbf{C} \quad \text { by } \quad \mathbf{F}_{\jmath} \equiv \Delta\left(\lambda_{j}^{c}(\vec{q}) ; \vec{q}\right),
$$

together with the equivalent sequence $\mathbf{G}_{j}$ as defined immediately below Eq. (3.1). The functionals $\mathbf{G}_{j},|j| \leq N$, inherit the multivaluedness of the ordering. For $\vec{q} \in \mathscr{B}^{(h)}$, the sequence $\left\{j\left[\mathbf{G}_{j}(\vec{q})-2\right]\right\} \in l^{2}$. In fact, the decay in $j$ is even faster.

Note that the definition $\mathscr{B}^{(h)}$ does not explicitly show that it consists only in functions $\vec{q}(x) \in C^{\infty}$, for which the terms in the sequence $\left\{\mathbf{G}_{j}\right\}$ decay faster than any polynomial. We further restrict: Fix a sequence of positive numbers $\left\{\delta_{j}\right\}_{|j|>N}$ which decay faster than any polynomial as $|j| \rightarrow \infty$. Then we use the sequence of constants of motion, $\left\{\mathbf{G}_{\mathbf{j}}\right\}_{|\mathbf{j}|>\mathbf{N}}$, as ordered by the counting lemma, to define a smaller invariant set:

$$
\mathscr{\beta}^{(h, \delta)} \equiv\left\{\vec{q} \in \mathscr{R}^{(h)}\left|0 \leq\left[\left|\mathbf{G}_{j}(\vec{q})-2\right|\right] \leq \delta_{j} \forall\right| j \mid<N^{(h)}\right\} .
$$

$\mathscr{B}^{(h, \delta)} \subset \mathscr{F}$ is a closed, bounded, invariant set with boundary. Since $\mathscr{B}^{h, \delta)} \subset \mathscr{B}^{(h)}$, the counting lemma still applies. Moreover, $\mathscr{B}^{(h, \delta)}$ is compact in $C^{k}$.

Our restricted initial goal is to stratify $\mathscr{B}^{(h, \delta)}$ by the level sets of $\hat{L}$. Our strategy will be to use the entire family $\left\{\mathbf{G}_{j}, j=-\infty, \ldots,+\infty\right\}$ of constants of motion, together with the spectral theory of $\hat{L}$, to describe this stratification. Next, we define a critical function:

Definition 1. A function $\vec{q}_{*} \in \mathscr{乃}^{(h, \delta)}$ is critical if

1. $\vec{q}_{*} \in \partial \mathscr{B}^{(h, \delta)}$, or

2. $\vec{q}_{*}$ is a branch point; or 
3. on a fixed sheet, away from branch points, members of the set $\left\{\operatorname{grad} \mathbf{G}_{j}\right\}_{j=-\infty}^{+\infty}$ are linearly dependent at $\vec{q}_{*}$.

Several remarks are appropriate:

Remark 1 . Type 1 critical functions are artificial in that the boundary $\partial \mathscr{B}^{(h, \delta)}$ is artificial. We will not consider them further.

Remark 2. $\vec{q}_{*}$ is a type 2 critical function for $\mathbf{G}_{j} \Leftrightarrow$ $\Delta^{\prime}\left(\lambda_{j}^{c}\left(\vec{q}_{*}\right) ; \vec{q}_{*}\right)=\Delta^{\prime \prime}\left(\lambda_{j}^{c}\left(\vec{q}_{*}\right) ; \vec{q}_{*}\right)=0$.

Remark $3 \vec{q}_{*}$ is a type 3 , but not type 2 , critical function for $\mathbf{G}_{j} \Leftrightarrow \exists$ at least one $j$ such that, at $\vec{q}=\vec{q}_{*}$,

$$
\operatorname{grad} \mathbf{G}_{j}=0 ; \text { that is } \mathbf{F}_{j}= \pm 2 \text { and } \mu_{j}=\lambda_{j}^{d} .
$$

Therefore, for type 3 critical functions, it is sufficient to focus attention upon the condition $\operatorname{grad} \mathbf{G}_{j}=0$.

Remark 4. The type 3 critical functions cannot be isolated points in $\mathscr{F}$ unless $\operatorname{grad} \mathbf{G}_{3}=0 \forall j$. Moreover, by a version of "Borg's Theorem" for the Zakharov-

Shabat operator $\hat{L}$, the only function for which all of these gradients vanish is the point $\vec{q}_{*}=\overrightarrow{0}$. The reason that critical functions cannot be isolated is that the functionals $\left\{\mathbf{G}_{j} \forall j\right\}$ constitute a family of commuting constants of motion for the NLS Hamiltonian system. Given a critical function $\vec{q}_{*}$ for which $\operatorname{grad} \mathbf{G}_{l}=0$ while $\operatorname{grad} \mathbf{G}_{k} \neq 0$, one can use $\mathbf{G}_{k}$ as a Hamiltonian with which to map the original critical function $\vec{q}_{*}$ to $\vec{q}_{*}\left(\tau_{k}\right)$. The functions $\vec{q}_{*}\left(\tau_{k}\right)$ provide a continuous one parameter family (indexed by the flow parameter $\tau_{k}$ ) of distinct critical functions for which $\operatorname{grad} \mathbf{G}_{l}=0$. At $\tau_{k}=0, \vec{q}_{*}\left(\tau_{k}\right)=\vec{q}_{*}$; the original critical function is certainly not isolated!

Thus, critical functions of a commuting family of constants of motion are rarely isolated. In order to develop a Morse theory for integrable systems, one must study manifolds of critical functions for an entire family $\left\{\mathbf{G}_{j}, \forall j\right\}$ of constants of motion. One approach to such a study in finite dimensions was introduced Bott $[3,5]$, and later adapted to Hamiltonian systems by Fomenko [11]. This theory begins with the notion of a "Bott function:"

Definition 2. Let $F$ be a real valued, differentiable function on a differentiable Hilbert manifold $\mathscr{l}$. A connected submanifold $\mathscr{V}$ of $\mathscr{C}$ is called a non-degenerate critical manifold of $F$, if

1. Every point $q \in \mathscr{V}$ is a critical point of $F$; that is, $\left.\operatorname{grad} F\right|_{q}=0$.

2. For each point $q \in \mathscr{V}$, the null space of the Hessian of $F$ is precisely the tangent space to $\mathscr{V}$.

A function $F$ which possesses such a critical manifold is call a Bott Function.

This definition is a natural extension to an infinite dimensional setting of a definition introduced in [4] for finite dimensions. It immediately applies outside of the disc $D$.

\subsection{Outside the Disc $D:|j|>N$}

In this subsection, we consider $\mathbf{G}_{j},|j|>N$. By the counting lemma, the critical points $\left\{\lambda_{j}^{c},|j|>N\right\}$ are real and simple; $\Delta^{\prime \prime}\left(\lambda^{c}\right) \neq 0$. Hence, there are not type 2 

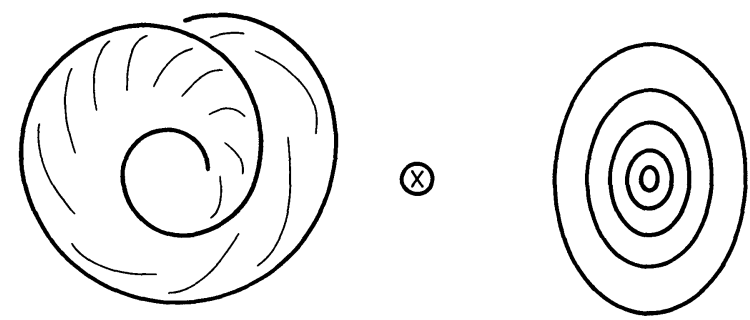

Fig. 5.1. The level set of $\mathbf{G}_{j}$, fixed $|j|>N$. (The value of $\mathbf{G}_{j}$ fixes the radii of the circles which comprise the disc on the right)

critical functions associated with these critical points outside the disc $D$. Each $\mathbf{G}_{\mathbf{j}}$ is maximal, with maximal value of 2 , at its critical potentials. A consequence is that, for each $j,|j|>N$, for each value of $\mathbf{G}_{\mathbf{j}},\left[2-\mathbf{G}_{\mathbf{j}}\right]=\delta_{\mathbf{j}}^{*}$, the level set is topologically a circle. Moreover, as $\delta_{j}^{*}$ runs over $\left[0, \delta_{j}\right]$, this circle fills out a closed disc, as is depicted in Fig. 5.1.

To describe this situation, we first set $\delta_{j}^{*}=0 \forall|j|>N$. In this case, inverse spectral theory guarantees that $\mathscr{B}^{(h, \delta)}$ is a manifold $\mathscr{B}_{N}^{h}$ of dimension [2(N+1)]:

$$
\mathscr{P}_{N}^{h} \equiv \mathscr{B}^{\left(h ; \overrightarrow{0}, \delta_{-N}, \quad, \delta_{N}, \overrightarrow{0}\right)}
$$

For a fixed $\delta_{j}=\delta_{j}^{*}(\neq 0),|j|>N, \mathscr{B}^{\left(h, \delta^{*}\right)}$ is topologically a direct product of $\mathscr{B}_{N}^{h}$ with discs $D_{j}$, one for each $|j|>N$.

This fact can be restated in the standard language of Morse theory: We define the single real valued functional

$$
\begin{gathered}
\mathbf{G}: \mathscr{B}^{(h, \delta)} \rightarrow \mathbf{R}, \\
\mathbf{G} \equiv \sum_{|j|>N} \delta_{j}^{-1}\left[2-\mathbf{G}_{j}\right] .
\end{gathered}
$$

Theorem 4. (1) $\left.\operatorname{grad} \mathbf{G}\right|_{q_{*}}=0 \Leftrightarrow \vec{q}_{*} \in \mathscr{B}_{N}^{h}$;

(2) $\mathbf{G}$ is a Bott function;

(3) The gradient flow for $\mathbf{G}$ shows that $\mathscr{B}^{(h, \delta)}$ is homotopic to $\mathscr{B}_{N}^{h}$; i.e., $\mathscr{B}_{N}^{h}$ is a deformation retract of $\mathscr{B}^{(h, \delta)}$.

Remark. The functional $\mathbf{G}$ controls the smoothness of $\vec{q}$ because it controls the tail of $\left\{\mathbf{F}_{j}\right\}$. However, it provides no control of $\left\{\mathbf{F}_{j}\right\}$ for $|j| \leq N$; thus, mollifiers must be used to guarantee that the gradient flow for $\mathbf{G}$ is well defined on $\mathscr{B}^{(h, \delta)}$.

The proof of this theorem is only a reformulation of the spectral material described above, once one uses the representation of $\operatorname{grad} \mathbf{G}$ in terms of "squared eigenfunctions." Part (1) follows from the linear independence of these "squares."

To summarize, that part of the stratification associated with the infinite dimensional tail $|j|>N$ consists in nested tori, just as in the KdV case with selfadjoint $\hat{L}$. Thus, it is sufficient to restrict attention to the $[2(N+1)]$ dimensional invariant set $\mathscr{B}_{N}^{h}$. 

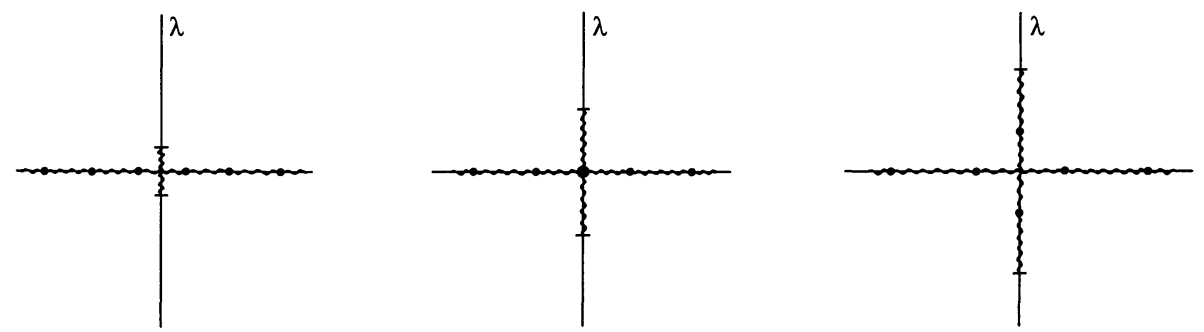

Fig. 5.2. First spectral transition on the invariant plane $\pi$

\subsection{Inside the Disc $D: \mathbf{F}_{\mathbf{j}},|\mathbf{j}| \leq \mathbf{N}$}

The invariant set $\mathscr{B}_{N}^{h}$, as defined by setting $\mathbf{G}_{j}=2,|j|>N$, is a finite dimensional invariant manifold with boundary, which is closed, bounded, and compact. To describe its stratification by level sets, one can restrict attention to the constants $\left\{\mathbf{G}_{j}\right\}_{|j| \leq N}$.

Inside the disc $D$, critical points $\lambda^{c}$ can exist for which $\Delta^{\prime \prime}\left(\lambda^{c}\right)=0$; that is, type 2 critical functions can exist. On the other hand, away from branch points, one can estimate the number $N_{D}$ of linearly independent members of the set $\left\{\operatorname{grad} \mathbf{G}_{j}\right\}_{|j| \leq N}$ as follows:

$$
2\left(N-N_{d}\right)+1 \leq N_{D} \leq 2 N+1 \quad N_{D}=2\left(N-N_{d}^{\mu}\right),
$$

where $N_{d}=$ the number of double points in the upper half complex plane, counted according to multiplicity, and $N_{d}^{\mu}=$ the number of upper half plane double points with a Dirichlet $\mu$ locked to the double point.

In contrast to the simple behavior associated with $|j|>N$, very complicated homotopic behavior can be associated to $\mathbf{G}_{j},-N \leq j \leq N$, which in turn is associated to passage through critical spectral configurations within the disc $D$. Of particular importance will be the colliding of critical points $\lambda_{j}^{c}$ and transition values at which $\lambda_{j}^{c}$ changes from real to complex. Both of these transitions are associated with type 2 critical functions.

A global Morse theory, even for $\mathscr{S}_{N}^{h}$, seems very difficult to us, primarily because of the difficulty in labeling all possible critical spectral configurations within the disc $D$; it is certainly beyond our reach at this time. The following examples illustrate concretely the difficulties which arise; yet, we believe that they also indicate the potential power of a method which uses spectral theory to implement a Morse description of the level sets.

5.2.1. Example 1: Near an Invariant Whisker. Let $I$ denote the plane of constants,

$$
\Pi \equiv\left\{\vec{q} \in \mathscr{F} \mid \partial_{x} \vec{q}=\overrightarrow{0}\right\},
$$

and, setting $N=1$, we define the disc $\mathscr{D}^{(h)} \subset \Pi$ by

$$
\mathscr{D}^{(h)} \equiv \Pi \cap \mathscr{B}_{1}^{h} .
$$

The spectrum of $\hat{L}(\vec{q})$, for $\vec{q} \in \mathscr{D}^{(h)}$, consists in the real axis union a single band on the imaginary axis. We choose the parameter $h$ large enough that two complex double points (conjugates of each other) can exist on this band of purely imaginary spectrum, but small enough that no more than two can exist. Figure 5.2 depicts the two classes of spectral configurations, together with an important critical configuration. 


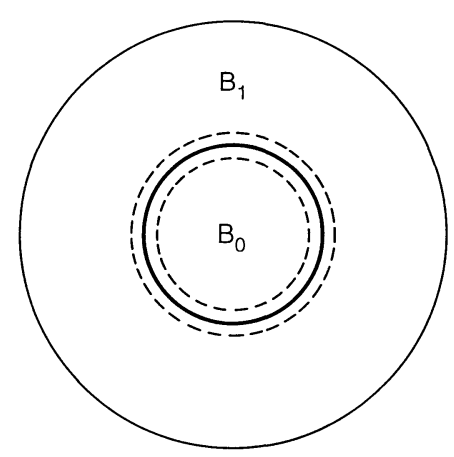

Fig. 5.3. The open annuli $B_{0}$ and $B_{1}$ which do not contain the critical circle
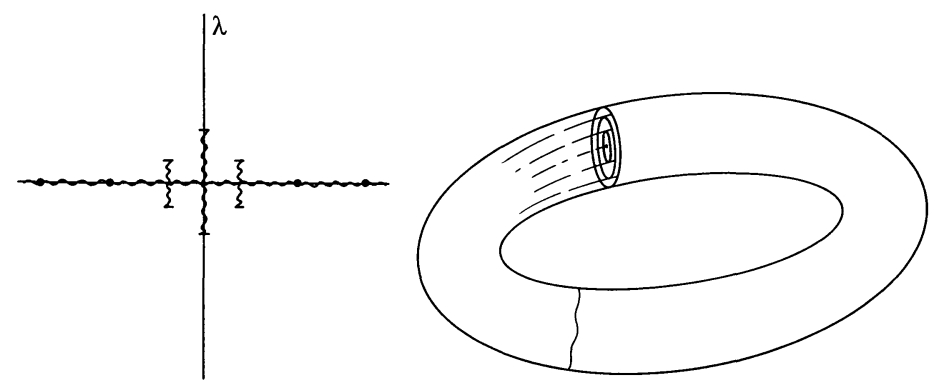

Fig. 5.4. Spectral configuration and level set foliation of real Morse function $G_{1}$ for real critical points inside the disc

[The origin is always a critical point. Typically, it is simple $\left(\Delta^{\prime \prime} \neq 0\right)$; at the critical configuration, is is double $\left(\Delta^{\prime \prime}=0\right)$.]

Potentials in $\mathscr{D}^{(h)}$ which have this critical spectral configuration consist in a circle in $\mathscr{D}^{(h)},|q|=r_{c}$, which divides $\mathscr{D}^{(h)}$ into two annuli $\mathscr{A}_{0}$ and $\mathscr{C}_{1}$ :

$$
\mathscr{A}_{0}: 0 \leq|q| \leq r_{c}, \quad \mathscr{A}_{1}: r_{c} \leq|q| \leq r_{\max } .
$$

We also introduce two smaller open annuli $\mathscr{B}_{0}$ and $\mathscr{B}_{1}$ which do not contain the critical circle. (See Fig. 5.3).

Since $N=1$, the manifold $\mathscr{B}_{1}^{h}$ is six dimensional. We introduce two six dimensional neighborhoods (open cylinders) in $\mathscr{B}_{1}^{h}, \tilde{U}_{0}$ and $\tilde{U}_{1}$, containing the annuli $\mathscr{C}_{0}$ and $\mathscr{A}_{1}$, respectively. The spectrum of $\hat{L}(\vec{q})$ for even $\vec{q} \in \tilde{U}_{0}$, is shown in Fig. 5.4, and for even $\vec{q} \in \tilde{U}_{1}$, in Fig. 5.5. Notice that there are two distinct types of spectrum for $\vec{q} \in \tilde{U}_{1}$. These two types are important in the following.

5 2.2. Even Functions. First, we dispence with $\tilde{U}_{0}$, which is trivial and analogous to the behavior associated to $|j|>N$. Rather than treat the general case, we focus upon the submanifold of functions which are even about $x=0$, i.e. $\vec{q}(-x)=\vec{q}(x)$. This submanifold $\mathscr{E}_{1}^{h} \subset \mathscr{B}_{1}^{h}$ is four dimensional and plays an important role throughout our studies. The condition of evenness leads us to consider the constraints

$$
\begin{gathered}
\mathbf{G}_{0}=g_{0}, \quad g_{0} \in(-2,2), \quad \text { fixed }, \\
\mathbf{G}_{-1}=\mathbf{G}_{1}, \quad \mathbf{G}_{1} \text { free. }
\end{gathered}
$$




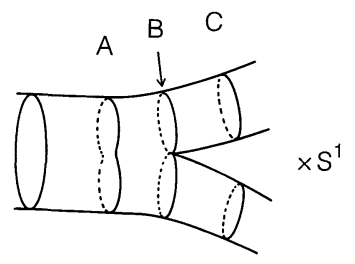

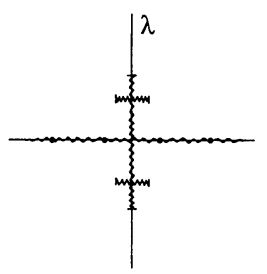

A

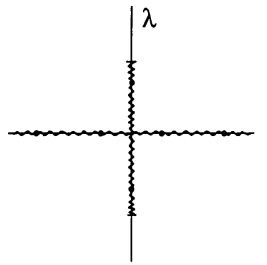

B

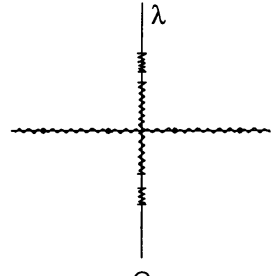

C

Fig. 5.5 The Trouser for even potentials and its spectral identification

In this case, the critical value of $\mathbf{G}_{1}$ is -2 , which the Hessian shows is a minimum. The critical level set is a circle containing only functions on the plane of constants $\Pi$. As $\mathbf{G}_{1}$ increases from its critical value -2 , the neighboring level sets in $\mathscr{E}_{1}^{h}$ are two tori. The spectral configurations, together with a schematic of the level sets, are depicted in Fig. 5.4. Thus, this trivial case of $\tilde{U}_{0}$ is essentially the same as that for real critical points outside the disc $D$.

Turning to the more interesting case of $\tilde{U}_{1}$, we continue with even functions, $\mathscr{E}_{1}^{h} \cap \tilde{U}_{1}$, for which the complex double point $\lambda_{1}^{d}$ lies in the band of spectrum on the imaginary axis, and $\mathbf{G}_{-1}$ vanishes identically. We are left with two independent constants of motions, $\mathbf{G}_{0}$ and $\mathbf{G}_{1}$. We define a submanifold by setting $\mathbf{G}_{0}=g_{0}$, $g_{0} \in(-2.2), g_{0}$ fixed. This submanifold is three dimensional, lying within $\mathscr{E}_{1}^{h} \cap \tilde{U}_{1}$. For our choice of $g_{0}, \mathbf{G}_{0}$ has no type 2 or type 3 critical points on this submanifold. We intend to view $\mathbf{G}_{1}$, restricted to this submanifold, as a Bott function.

In this even case, the critical value of $\mathbf{G}_{1}$ is -2 , and the critical set is once again a circle containing only functions on the plane of constants $\Pi$. The Hessian is a $3 \times 3$ matrix with one positive, one negative, and one zero eigenvalue. Its null vector is tangent to the critical circle; the saddle structure which exists since the Hessian has index 1 shows that this circle is a "whiskered circle."

Next, we use Bäcklund transformations to construct an explicit representation of this whiskered circle; that is, to construct the critical level set. In the even case, the Bäcklund formula 4.11

$$
\begin{aligned}
Q_{H}= & {\left[\frac{\cos 2 p-\sin p \operatorname{sech} \tau \cos (2 k x+\phi)-i \sin 2 p \tanh \tau}{1+\sin p \operatorname{sech} \tau \cos (2 k x+\phi)}\right] } \\
& \times c e^{-i\left(2 c^{2} t+\gamma\right)} \rightarrow e^{\mp 2 i p} c e^{-i\left(2 c^{2} t+\gamma\right)} \text { as } \varrho \rightarrow \mp \infty
\end{aligned}
$$

must be specialized in order to ensure evenness by one of two choices of $\phi: \phi=0$, $\pi$. There are two disjoint whiskers, one for each choice of $\phi$; each whisker is two dimensional, parameterized by $\varrho$ and $\gamma$. For fixed $\gamma$, a "figure eight" structure appears. Orbits on the critical level set are homoclinic to the critical circle, which is also parameterized by $\gamma$. Note the phase shift of $4 p$ experienced by these homoclinic orbits. 


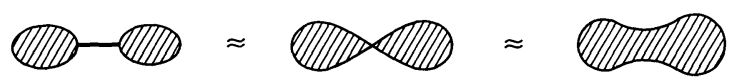

Fig. 5.6. Homotopy transition for even potentials

A neighborhood of the critical level set in this even setting is depicted as a "trouser" in Fig. 5.5. The Bäcklund formula explicitly shows that one leg of the trouser is associated with a spatial excitation located near $x=0$, while the other leg is associated with an excitation located near $x=1 / 2$. This fact plays a central role in the interpretation of chaotic behavior which arises when the integrable system is perturbed [27].

In this even setting, the "trouser" can be described in the language of Morse (Bott) theory. In order to discuss the homotopy transitions, we define

$$
M^{c} \equiv\left\{\vec{q} \in \mathscr{E}_{1}^{h} \cap \tilde{U}_{1} \mid \mathbf{G}_{1} \leq c\right\},
$$

then

$$
\begin{aligned}
M^{-2-\varepsilon} & =2 D \times S^{1} \\
M^{-2} & =\hat{D} \times S^{1} \\
M^{-2+\varepsilon} & =D \times S^{1} .
\end{aligned}
$$

Here $\varepsilon$ is sufficiently small, $D$ denotes a two-disc, and $2 D$ denotes two disjoint two discs. $\hat{D}$ denotes a two-disc with two points on its boundary identified. The homotopy transition formula is then given by

$$
D \times S^{1} \approx \hat{D} \times S^{1}=\left[2 D \times S^{1} \cup e^{1}\right] .
$$

Here $\cup$ represents cell attachment and $e^{1}$ represents a 1-cell (see Fig. 5.6). A 1-cell is attached because the Morse index of $\mathbf{G}_{1}$ is 1 .

5.2.3 No Spatial Symmetry. Next, we remove the constraint of evenness. In this case, there are three independent constants of motion, $\left\{\mathbf{G}_{-1}, \mathbf{G}_{0}, \mathbf{G}_{1}\right\}$. We are tempted to freeze $\mathbf{G}_{-1}=g_{-1}$ and $\mathbf{G}_{0}=g_{0}$, and to use $\mathbf{G}_{1}$ as a Bott function; however, unless the value $g_{-1}=0, \mathbf{G}_{1}$ has no critical functions. Moreover, at the level $g_{-1}=0, \mathbf{G}_{-1}$ necessarily has a critical function whenever $\mathbf{G}_{1}$ does. (See Lemma 7 of Sect. 3.) The upshot is that one must consider the pair $\mathbf{G}_{-1}$ and $\mathbf{G}_{1}$ simultaneously. (This situation necessarily extends the Fomenko strategy [11].)

We begin by considering

$$
\mathbf{G}_{1}: \tilde{U}_{1} \rightarrow \mathbf{R}
$$

The critical value of $\mathbf{G}_{1}$ is -2 , and the critical set is once again a circle containing only functions on the plane of constants $\Pi$. The Hessian has 2 positive and 2 negative eigenvalues, showing the existence of a saddle structure.

At a critical function, the value of $\mathbf{G}_{1}$ is -2 , and the value of $\mathbf{G}_{-1}$ is necessarily 0 . Freezing $\mathbf{G}_{0}$ at value $g_{0}$, we use Bäcklund transformations to construct explicitly the critical level set

$$
\begin{aligned}
\mathbf{G}_{0} & =g_{0}, \quad g_{0} \in(-2,2), \quad \text { fixed } \\
\mathbf{G}_{-1} & =0 \\
\mathbf{G}_{1} & =-2 .
\end{aligned}
$$




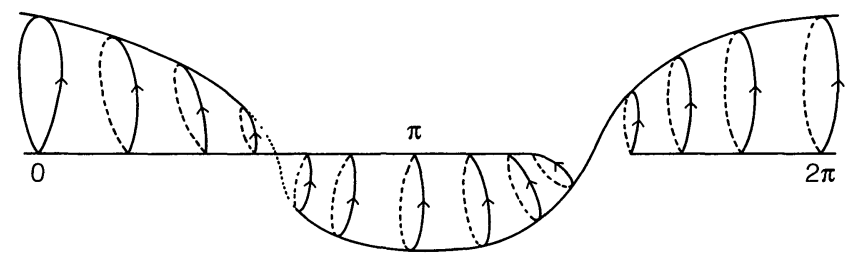

Fig. 5.7a. The construction of the whisker by identifying $\phi$ with $\phi+\pi$

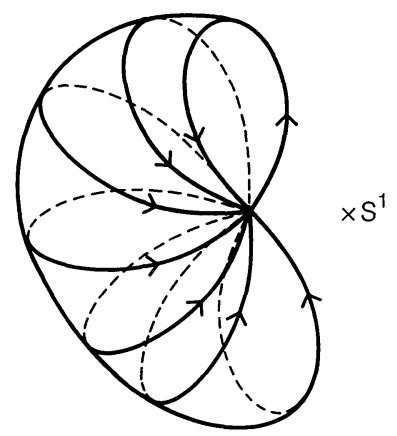

Fig. 5.7b. Half of the whole whisker (note the twist)

For convenience, we again repeat the Bäcklund formula:

$$
\begin{aligned}
Q_{H}= & {\left[\frac{\cos 2 p-\sin p \operatorname{sech} \tau \cos (2 k x+\phi)-i \sin 2 p \tanh \tau}{1+\sin p \operatorname{sech} \tau \cos (2 k x+\phi)}\right] } \\
& \times c e^{-\imath\left(2 c^{2} t+\gamma\right)} \rightarrow e^{\mp 2 i p} c e^{-i\left(2 c^{2} t+\gamma\right)} \text { as } \varrho \rightarrow \mp \infty
\end{aligned}
$$

The situation is depicted in Fig. 5.7.

The critical level set is constructed from a family of "figure eights," one for each value of the angle $\phi$. The Bäcklund formula shows that the critical level set is parameterized by $\varrho \in(0,+\infty), \phi \in[0,2 \pi)$, and $\gamma \in[0,2 \pi)$; together with the boundaries $\varrho=0, \varrho=+\infty$, each of which consists in a circle parameterized by $\phi \in[0,2 \pi)$. Realizing that all points on the horizontal $(\phi)$ axis of Fig. 5.7a are actually identified, one is lead to Fig. 5.7b, which depicts the level set as $S \times \hat{S}^{2}$, where $\hat{S}^{2}$ denotes a two-sphere with two points identified, together with a twist as is seen to be required by examining Fig. 5.7b. The critical level set is connected; we have already seen that constraints such as evenness can disconnect it.

In order to describe a neighborhood of the critical level set, we next consider the set

$$
\begin{aligned}
\mathbf{G}_{0} & =g_{0}, \quad g_{0} \in(-2,2), \quad \text { fixed }, \\
\mathbf{G}_{-1} & =0, \\
\mathbf{G}_{1} & =g_{1}, \quad g_{1} \in(-2-\varepsilon,-2+\varepsilon), \quad g_{1} \quad \text { free } .
\end{aligned}
$$

This set is not a manifold, but consists in manifolds of dimension 4 , together with boundary components. Selecting $g_{0}$ so that $\mathbf{G}_{0}$ has no critical points on the 4- 

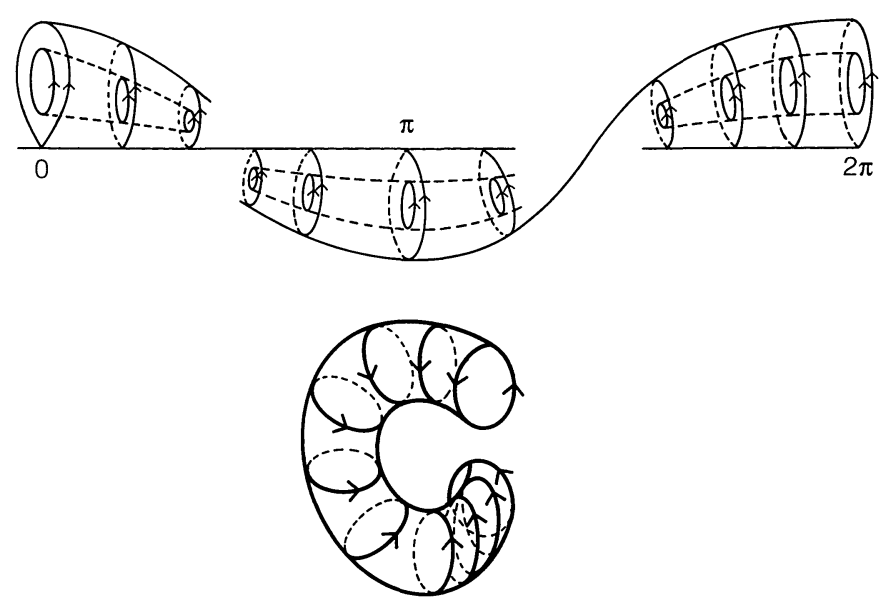

Fig. 5.8a. The level set for $g_{1}<-2$ (note the twist, half of the whole figure)
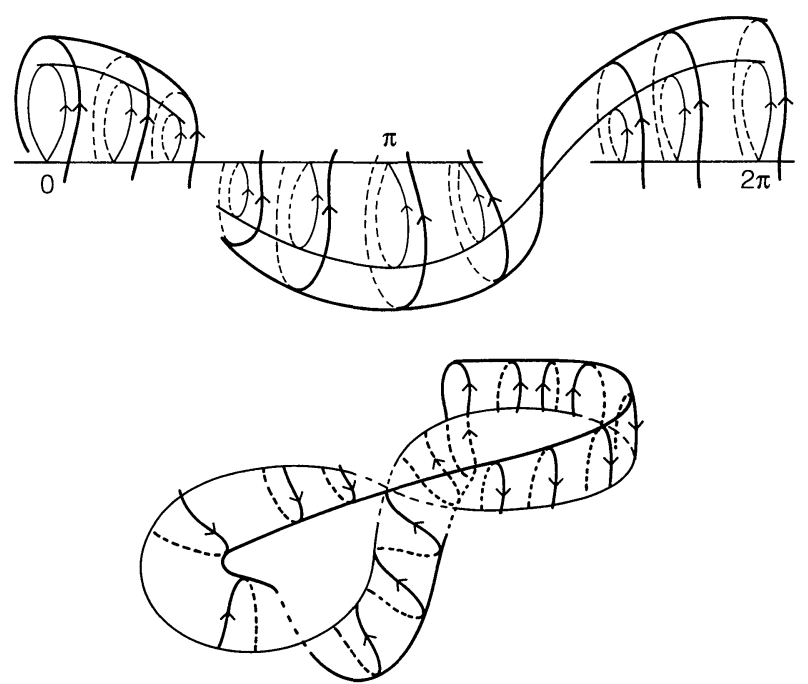

Fig. 5.8b. The level set for $g>-2$ (note the twist, half of the whole figure)

manifolds, one can view the 4-manifolds as three manifolds $\times S$. This situation is depicted in Figs. 5.8a and 5.8b.

To understand these figures, first fix $g_{1}<-2$. The spectrum of $\hat{L}$ has a gap on the imaginary axis, and the level set is a three torus $T^{3}$ as depicted. Note the twist. As $g_{1}<-2$ varies, one generates a nested family of three tori.

On the other hand, fix $g_{1}>-2$. The spectrum of $\hat{L}$ now has a cross on the imaginary axis. The level set remains a three torus $T^{3}$, but one looking very different as depicted. Again, note the twist.

In summary, as $\mathbf{G}_{1}$ varies near its critical value of -2 , the neighboring level sets are three tori, $T^{3}$. Moreover, each level set is connected. The three tori $T^{3}$ differ depending upon whether $\mathbf{G}_{1}$ is less than, or greater than, -2 . In particular, the three 


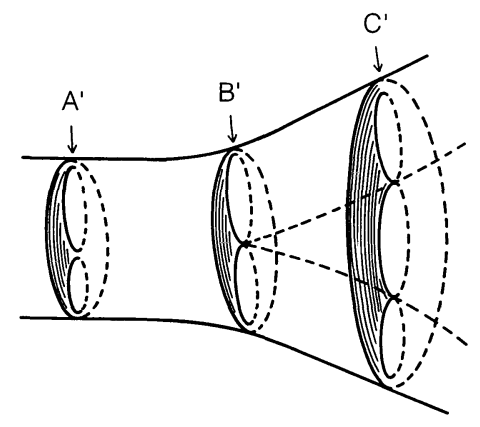

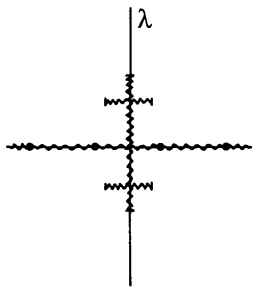

$A^{\prime}$

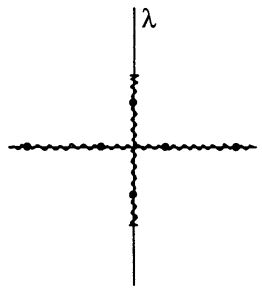

$B^{\prime}$

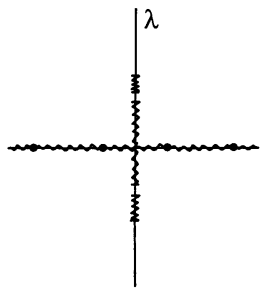

$C^{\prime}$

Fig. 5.8c. The exotic trouser and its spectral identification

tori have a twist. The consequences are that the level sets near the critical one appear as an "exotic trouser,"as depicted in Fig. 5.8c.

In an attempt to set up a Morse theory for these level sets, we begin with the set

$$
\begin{aligned}
\mathbf{G}_{0} & =g_{0}, \quad g_{0} \in(-2,2), \quad \text { fixed }, \\
\mathbf{G}_{-1} & =0, \\
\mathbf{G}_{1} & =g_{1}, \quad g_{1} \in(-2-\varepsilon,-2+\varepsilon), \quad g_{1} \quad \text { free. }
\end{aligned}
$$

Fomenko's strategy of using $\mathbf{G}_{1}$ as a Bott function will not work because

- this set is not a manifold;

- $\mathbf{G}_{-1}$ has critical functions in the set;

- the pair $\mathbf{G}_{-1}$ and $\mathbf{G}_{1}$ must be considered simultaneously.

Nevertheless, one can anticipate the homotopy transitions: Define

$$
M^{c} \equiv\left\{\vec{q} \in \tilde{U}_{1} \mid \mathbf{G}_{1} \leq c\right\}
$$

then

$$
\begin{aligned}
M^{-2-\varepsilon} & =\left(S^{1} \times D^{2}\right) \times S^{1} ; \\
M^{-2} & =\hat{B}^{3} \times S^{1} ; \\
M^{-2+\varepsilon} & =B^{3} \times S^{1} .
\end{aligned}
$$

Here $\varepsilon$ is sufficiently small, $\left(S^{1} \times D^{2}\right)$ is a solid torus, $\hat{B}^{3}$ is a 3 -ball with two points on its surface identified, and $B^{3}$ is a 3 -ball. The homotopy transition formula is then 


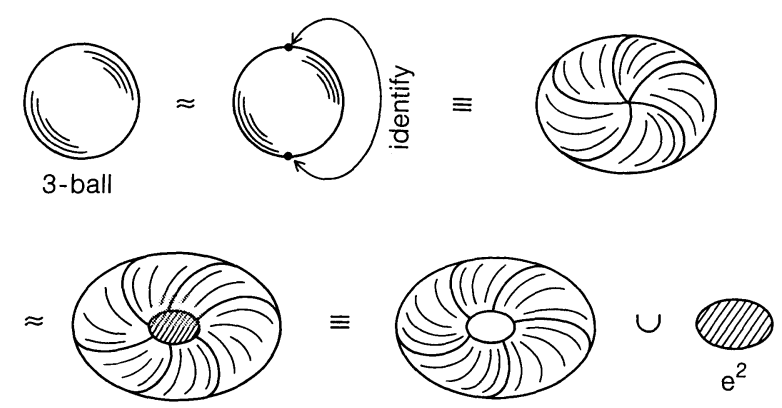

Fig. 5.9. Homotopy transitions without even constraints

given by

$$
B^{3} \times S^{1} \approx \hat{B}^{3} \times S^{1}=\left(S^{1} \times D^{2}\right) \times S^{1} \cup e^{2} .
$$

Here $\cup$ represents cell attachment and $e^{2}$ represents the 2-cell (see Fig. 5.9).

5.2.4. Higher Order Critical Points. This example, near the plane of constants, also contains type 2 critical behavior which occurs when $\Delta^{\prime \prime}\left(\lambda^{(c)} ; 0\right)=0$ and involves the collision of two or more critical points. Type 2 behavior can occur under even symmetry, and it has occurred (with both simple and rather complicated spectral configurations) in the numerical experiments of [27] which show temporally chaotic behavior of solutions of NLS under perturbations. Several explicit examples of type 2 spectral and level set behavior may be found in [20]. Limitations of space prevent us from presenting those examples here.

\subsection{Discussion}

As one begins to develop a Morse theory for the stratification of isospectral invariant sets for the NLS system, the first obstacle that one faces is the infinite dimensionality of the system. With the "counting lemmas" this difficulty is readily overcome. The infinite dimensional "tail" of the sequence of constants, $\left\{\mathbf{F}_{j}\right\}_{|j|>N}$, which is constructed from (necessarily real) critical points in the exterior of the disc $D$, is quite tame. Nothing emerges but nested tori, just as in the $\mathrm{KdV}$ case with its selfadjoint spectral problem.

The real obstacles to a global theory originate from the complicated spectral configurations for this nonselfadjoint spectral problem which can occur inside the disc $D$. Although these configurations are finite in number, they seem difficult to classify. Any classification or ordering will certainly depend upon the configurations of critical points of high order $\left[\lambda_{j}^{c}\right.$ such that $\Delta^{\prime \prime}\left(\lambda_{j}^{c}\right)=0$, etc.] Because of the presence of these higher order critical points, any Morse or Bott theory based upon the constants $\left\{\mathbf{F}_{j}\right\}$ seems necessarily local in function space. Our examples have displayed submanifolds, defined by freezing all constants but one, which possess critical functions for that one remaining constant for which the index is not invariant over the entire submanifold. Other examples show that type 2 critical functions, which are branch points for the functionals $\mathbf{F}_{\jmath}$, necessarily exist. These examples make it clear that to study such type 2 critical functions, several constants, $\left\{\mathbf{F}_{\jmath}\right\}$, for several values of $j$, must be studied simultaneously. In addition, in a general 
situation without additional constraints such as evenness, complex double points also require the simultaneous study of two constants, the real and imaginary parts of $\mathbf{F}$. Such vector valued Bott functions take one beyond classical Bott theory and pose challenging mathematical problems.

\section{A Melnikov Vector}

When studying perturbations of integrable systems, one frequently begins with whiskered tori for the integrable system and asks about their persistence under perturbations. If the lower dimensional tori persist, hyperbolic structure such as the stable and unstable manifolds of the tori persists as well. In the integrable case the stable and unstable manifolds coincide, guaranteeing the existence of orbits in the integrable system which are homoclinic to the low dimensional critical tori. Under perturbations, these stable and unstable manifolds split apart; typically homoclinic orbits do not persist. If, however, the perturbed stable manifold intersects transversety the perturbed unstable manifold, orbits homoclinic to the persistant tori will exist. One method which is commonly used to measure the splitting of stable and unstable manifolds is that of Melnikov [32], which uses "Melnikov functions" to provide estimates of the distance between these manifolds. These Melnikov methods are particularly natural tools with which to prove existence of homoclinic orbits in perturbations of integrable systems.

The detailed geometry of these transverse intersections differs from system to system, and from case to case; it is certainly beyond the scope of this article to describe this geometry in any detail. Examples in the framework of soliton systems may be found in $[18,27,31,25,21]$. Here we simply note that in all cases, the Melnikov function is based upon the projection of the perturbed vector field (which defines the perturbed dynamical system) onto the gradient of an unperturbed constant of motion. Our point here is that the constants $\left\{\mathbf{F}_{j}\right\}$, together with Bäcklund transformations, provide natural representations of Melnikov vectors for perturbed soliton pde's.

We begin with a whiskered torus as represented by the Bäcklund formulas of Theorem 4. First, we consider the case of one instability associated with a complex double point $\nu$, for which the homoclinic orbit on the whisker is given by Bäcklund formula (4.5),

$$
Q_{H}(x, t) \equiv q(x, t)+2(\nu-\bar{\nu}) \frac{\phi_{1} \bar{\phi}_{2}}{\phi_{1} \bar{\phi}_{1}+\phi_{2} \bar{\phi}_{2}},
$$

where $\vec{q}$ lies on the critical lower dimensional torus and $\vec{\phi}$ denotes a general eigenfunction of $\hat{L}$ at $(\vec{q}, \nu)$, which, when expressed in terms of the Floquet basis $\left\{\vec{\psi}^{(+)}, \vec{\psi}^{(-)}\right\}$, takes the form

$$
\bar{\phi}=c_{+} \vec{\psi}^{(+)}+c_{-} \vec{\psi}^{(-)} .
$$

Next, we consider a perturbation of the NLS equation generated by a perturbed vector field $\varepsilon f(\vec{q})$. In this setting the Melnikov function can be defined using the constant $\left\{\mathbf{F}_{j}\right\}$, where $\lambda_{j}^{c}=\nu$ :

$$
\mathbf{M}_{\mathbf{j}} \equiv \int_{-\infty}^{+\infty}\left[\left.\left\langle\operatorname{grad} \mathbf{F}_{j}, \bar{f}\right\rangle\right|_{q=Q}\right] d t
$$

where the integrand is evaluated along the unperturbed homoclinic orbit $\vec{q}=\vec{Q}_{H}$. 
This integrand can be expressed rather explicitly using Bäcklund transformations. We begin with Eq. (3.2) for the $\operatorname{grad} \mathbf{F}_{j}$,

$$
\frac{\delta \mathbf{F}_{j}}{\delta \vec{q}}=-i \frac{\sqrt{\Delta^{2}-4}}{W\left[\Psi^{(+)}, \Psi^{(-)}\right]}\left(\begin{array}{l}
\Psi_{2}^{(+)} \Psi_{2}^{(-)} \\
\Psi_{1}^{(+)} \Psi_{1}^{(-)}
\end{array}\right),
$$

where $\vec{\Psi}^{ \pm}$are a Floquet basis at $\left(\vec{Q}_{H}, \nu\right)$. This Floquet basis can be obtained from Bäcklund formula (4.6):

$$
\vec{\Psi}^{( \pm)}(x, t ; \lambda) \equiv G(\lambda ; \nu ; \vec{\phi}) \vec{\psi}^{( \pm)}(x, t ; \lambda),
$$

with the transformation matrix $G$ given in terms of the general eigenfunction $\vec{\phi}$ at $(\vec{q}, \nu)$ by

$$
\begin{aligned}
G=G(\lambda ; \nu ; \vec{\phi}) & \equiv N\left(\begin{array}{cc}
\lambda-\nu & 0 \\
0 & \lambda-\bar{\nu}
\end{array}\right) N^{-1}, \\
N & \equiv\left[\begin{array}{cc}
\phi_{1} & -\bar{\phi}_{2} \\
\phi_{2} & \bar{\phi}_{1}
\end{array}\right] .
\end{aligned}
$$

These Bäcklund formulas are rather easy to manipulate to obtain explicit information. For example, the transformation matrix $G(\lambda, \nu)$ has a simple limit as $\lambda \rightarrow \nu$ :

$$
\lim _{\lambda \rightarrow \nu} G(\lambda, \nu)=\frac{\nu-\bar{\nu}}{|\vec{\phi}|^{2}}\left(\begin{array}{cc}
\phi_{2} \bar{\phi}_{2} & -\phi_{1} \bar{\phi}_{2} \\
-\phi_{2} \bar{\phi}_{1} & \phi_{1} \bar{\phi}_{1}
\end{array}\right)
$$

where $|\vec{\phi}|^{2}$ is defined by

$$
|\vec{\phi}|^{2} \equiv \phi_{1} \bar{\phi}_{1}+\phi_{2} \bar{\phi}_{2}
$$

With formula (6.3) one quickly calculates

$$
\begin{aligned}
\lim _{\lambda \rightarrow \nu} \vec{\Psi}^{( \pm)} & =\lim _{\lambda \rightarrow \nu} G \vec{\psi}^{( \pm)} \\
& = \pm c_{\mp} W\left[\vec{\psi}^{(+)}, \vec{\psi}^{(-)}\right] \frac{\nu-\bar{\nu}}{|\vec{\phi}|^{2}}\left(\begin{array}{c}
\bar{\phi}_{2} \\
-\bar{\phi}_{1}
\end{array}\right)
\end{aligned}
$$

from which one sees that $\vec{\Psi}^{(+)}$and $\vec{\Psi}^{(-)}$are dependent at $\left(\vec{Q}_{H}, \lambda=\nu\right)$,

$$
\vec{\Psi}^{(+)}=-\frac{c_{-}}{c_{+}} \vec{\Psi}^{(-)} .
$$

Remark. The dependence of $\vec{\Psi}^{(+)}$and $\vec{\Psi}^{(-)}$is required because, for $\vec{Q}_{H}$ on a whisker, the geometric multiplicity of $\nu$ is only one, even though its algebraic multiplicity is two or higher. Also, it is interesting to note that by manipulating the Bäcklund formula for $\vec{\Psi}^{( \pm)}$before taking the limit as $\lambda \rightarrow \nu$, one can obtain explicitly a second linearly independent solution of $\hat{L} \vec{\Psi}=\nu \vec{\Psi}$, but this second solution is not periodic in $x$.

Next, the Bäcklund formula gives a nice representation of the Wronskian

$$
W\left[\Psi^{(+)}, \Psi^{(-)}\right]=(\lambda-\nu)(\lambda-\bar{\nu}) W\left[\psi^{(+)}, \psi^{(-)}\right],
$$

from which the following limit is easy to compute:

$$
\lim _{\lambda \rightarrow \nu} \frac{\sqrt{\Delta^{2}-4}}{W\left[\Psi^{(+)}, \Psi^{(-)}\right]}=\frac{\sqrt{\Delta(\nu) \Delta^{\prime \prime}(\nu)}}{(\nu-\bar{\nu}) W\left[\psi^{(+)}, \psi^{(-)}\right]} .
$$


With formulas (6.2-6.4), one obtains the explicit representation of the $\operatorname{grad} \mathbf{F}_{j}$ :

$$
\frac{\delta \mathbf{F}_{j}}{\delta \vec{q}}=C_{\nu} \frac{c_{+} c_{-} W\left[\psi^{(+)}, \psi^{(-)}\right]}{|\vec{\phi}|^{4}}\left(\begin{array}{c}
\bar{\phi}_{1}^{2} \\
\bar{\phi}_{2}^{2}
\end{array}\right)
$$

where the constant $C_{\nu}$ is given by

$$
C_{\nu} \equiv i(\nu-\bar{\nu}) \sqrt{\Delta(\nu) \Delta^{\prime \prime}(\nu)} .
$$

Remark. Since $\vec{\phi}=c_{+} \vec{\psi}^{(+)}+c_{-} \vec{\psi}^{(-)}$, one sees explicitly from formula (6.5) that $\left.\frac{\delta \mathbf{F}_{j}}{\delta \vec{q}}\right|_{(Q, \nu)} \rightarrow 0$ as $c_{+} / c_{-} \rightarrow 0, \infty$. Also, since the eigenfunctions $\vec{\psi}^{(+)}$and $\vec{\psi}^{(-)}$at the complex double point $\nu$ grow or decay exponentially,

$$
\vec{\psi}^{( \pm)} \approx \exp \left( \pm \sigma_{\nu} t\right), \quad t \rightarrow \infty
$$

and the formula also shows explicitly that $\left.\operatorname{grad} \mathbf{F}_{j}\right|_{(Q, \nu)} \rightarrow 0$ as $t \rightarrow \infty$. The vector field $\operatorname{grad} \mathbf{F}_{j}$ must vanish because, in these limits, the point $\vec{Q}_{H}$ on the whisker tends to a critical function of $\mathbf{F}_{j}$.

Remark. This exponential decay in $t$ leads to convergence of the Melnikov integral When one uses other constants of motion to define the Melnikov integral, they need not be critical on the critical circle, the integrals need not converge, and a rather strange notion of conditional convergence must be developed [15]. It seems more natural to us to use constants which are critical on the "target" to define the Melnikov integrals in the first place.

With these ingredients, one obtains the following beautiful representation:

Theorem 5. The Melnikov function associated to the general complex double point $\nu$ admits the representation:

$$
\mathbf{M}_{j}=C_{\nu} c_{+} c_{-} \int_{-\infty}^{+\infty} \int_{0}^{1} W\left[\vec{\psi}^{(+)}, \vec{\psi}^{(-)}\right]\left[\frac{\left(\bar{\phi}_{1}^{2}\right) f_{1}(\vec{Q})+\left(\bar{\phi}_{2}^{2}\right) f_{2}(\vec{Q})}{|\vec{\phi}|^{4}}\right] d x d t .
$$

In the case of several complex double points, each associated with an instability, one can iterate the Bäcklund transformations and use those functionals $\mathbf{F}_{j}$ which are associated with each complex double point to obtain a representation of a Melnikov Vector.

A geometric interpretation of Melnikov function in the setting of damped driven perturbations of NLS will be given elsewhere $[21,20]$. Here we only emphasize that the constant $\mathbf{F}_{j}$ is natural for both Melnikov and Morse studies.

\section{Conclusion}

In this paper we have studied the focusing NLS equation (2.1) under periodic boundary conditions as a Hamiltonian system for functions $\vec{q} \in \mathscr{F}$. This NLS equation is completely integrable through the spectral transform for the Zakharov-Shabat linear operator $\hat{L}$. We have developed the nonselfadjoint Floquet spectral theory for the operator $\hat{L}$ in sufficient generality for later use in studies of perturbations of the NLS equation. 
Specifically, through $H^{1}$ counting lemmas we have shown that all eccentricities due to the nonselfadjointness of $\hat{L}$ are restricted to lie within a disc $D$ in the complex eigenvalue plane whose radius is controlled by the $H^{1}$ norm of $\vec{q}$. We then used these counting lemmas to introduce a natural local ordering of the spectrum and of a basis of $L^{2}$ which is constructed from "squared eigenfunctions," each member of which satisfies the linearized NLS equation. Obstructions to a global ordering for this basis were discussed which lead to an intriguing monodromy problem. This basis was then used to associate instabilities with complex multiple points in the spectrum of $\hat{L}$.

In this manner, critical spectral configurations were identified. Bäcklund transformations at these critical spectral configurations were then used to produce global representations of whiskered tori, together with proofs that the whiskeres are finite dimensional with estimates for their maximal dimension.

The operator $\hat{L}$ is not selfadjoint, making its Floquet spectral theory interesting. That spectral theory is studied through the $\lambda$ dependence of the Floquet discriminant

$$
\Delta: \mathbf{C} \times \mathscr{F} \rightarrow \mathbf{C},
$$

which is an NLS constant of motion for every value of $\lambda$. Through $\Delta$ we have introduced a natural sequence of constants of motion,

$$
\mathbf{F}_{j}(\vec{q}) \equiv \Delta\left[\lambda_{j}^{c}(\vec{q}) ; \vec{q}\right],
$$

where $\lambda_{j}^{c}$ denotes the $j^{\text {th }}$ critical point of $\Delta$, as ordered by the counting lemma. This sequence of constants $\left\{\mathbf{F}_{j}\right\}$ is useful for understanding both the spectrum of $\hat{L}$ and its isospectral level sets.

First, we computed the first and second variational derivatives of $\left\{\mathbf{F}_{j}\right\}$ in terms of squared eigenfunctions. The first variation vanishes at certain critical functions, which in turn are related to the critical spectral configurations. The Hessian at these critical functions provides information about the spectrum of $\hat{L}$. Specifically, it identifies allowed bifurcations in the spectrum at critical spectral configurations; thus, these local calculations identify interesting nonselfadjoint behavior such as crosses and gaps in the spectrum within the disc $D$, while confirming that only trivial behavior occurs outside that disc.

The Hessian also contains topological information about the isospectral level sets in the function space $\mathscr{F}$. The constant $\mathbf{F}_{j}$, as the height of $\Delta$ over the $j^{\text {th }}$ critical point, admits a natural interpretation as a Morse (or Bott) function. In Sect. 5 we have investigated this interpretation, showing that it is valid outside the disc $D$, and that it works well locally within the disc $D$. However, there are obstacles to a global Morse theory within the disc $D$. These obstacles occur because of the existence of higher order critical points $\lambda_{j}^{c}$ for which $\Delta^{\prime \prime}\left(\lambda_{j}^{c}\right)=0$; they are closely related to the monodromy problem for the global ordering mentioned above. Examples show that the resolution of these difficulties will require consideration of several of the constants simultaneously. Nevertheless, such Morse-like considerations do produce rather beautiful geometric examples of the behavior of the foliation near critical tori, as illustrated in the sketches in Sect. 5.

We emphasize, however, that the local bifurcation behavior of both the spectrum and the level sets, as successfully identified by the sequence of constants $\left\{\mathbf{F}_{j}\right\}$, is both adequate and sufficient for use in the analysis of the damped driven perturbation experiments surveyed in [27]. For example, the "trouser" diagram under even symmetry as discussed in Sect. 5.2.2 is central to one chaotic response of NLS to perturbations. Currently a study [17] including movie displays this perturbed chaotic 
behavior on an unperturbed background of "nested trousers." We hope that these examples will convince others to develop a global Morse description of NLS level sets.

Finally, we have remarked that the constants $\mathbf{F}_{j}$ are closely related to natural Melnikov functions which can be used for the analysis of perturbations of NLS. Bäcklund transformations provide general and explicit representations of these Melnikov functions, representations which converge precisely because the gradients of $\mathbf{F}_{j}$ vanish at the critical tori. Geometric interpretations of these Melnikov functions for specific examples in the NLS framework will be presented elsewhere.

Acknowledgements We gratefully acknowledge N. Ercolani, whose joint work [9] with one of us initiated our study of a Morse theory for the NLS equations, and who over the years has consistently advocated the use of spectral theory to implement Melnikov calculations. We also acknowledge Y. Chen, who helped with the early calculations of the Hessian, and several discussions with A. Soffer, J. Zhong, S. Wu, and W. Zhang concerning Sobolev arguments. Finally, we thank Eugene Trubowitz for reading an early manuscript. His suggested revisions have improved the work substantially.

\section{References}

1. Arnold, V I : Instabilities of systems with several degrees of freedom. Sov. Math. Dokl. 5, 581-585 (1964)

2. Bikbaev, R.F., Kuksin, S.B.: Algebra and analysis. No. 3, to appear 1992

3. Bott, R : Nondegenerate critical manifolds Ann. Math. 60, 248-261 (1954)

4 Bott, R.: Morse theory and its application to homotopy theory. 1960

5. Bott, R.: Lectures on morse theory old and new. Bull. AMS 7 (1982)

6. Ercolani, N.M., Forest, M.G.: The geometry of real sine-Gordon wavetrains. Commun. Math. Phys. 99, 1-49 (1985)

7 Ercolani, N.M., Forest, M.G., McLaughlin, D.W.: Geometry of the modulational instability. I, II. Preprint, University of Arizona, 1987

8 Ercolani, N M., Forest, M.G., McLaughlin, D.W.: Geometry of the modulational instability, Part III: Homoclinic orbits for the periodic sine-Gordon equation. Physica D 43, 349-384 (1990)

9. Ercolani, N.M., McLaughlin, D.W.: Toward a topological classification of integrable PDE's. The geometry of Hamiltonian systems. Berlin, Heidelberg, New York: Springer 1991

10 Flesch, R., Forest, M.G., Sinha, A : Numerical inverse spectral transform for the periodic sineGordon equation: theta function solutions and their linearized stability. Physica D 48, 169-231 (1991)

11. Fomenko, A.T.: Topological classification of all integrable Hamiltonian differential equations of general type with two degrees of freedom. Berlin, Heidelberg, New York: Springer 1991

12. Forest, M.G., McLaughlin, D.W.: Modulations of sine-Gordon and sinh-Gordon wavetrains. Stud. Appl. Math 68, 11-59 (1983)

13 Garnett, J., Trubowitz, E.: Gaps and bands of one dimensional periodic Schrödinger operators. Comment. Math. Helvetici, 59, 258-312 (1984)

14. Grebert, B., Guillot, J C.: Gaps of one dimensional periodic AKNS systems. Preprint, Ecole Polytechnique, 1990

15. Holmes, P.J., Marsden, J.E.: Horseshoes in perturbations of Hamiltonian systems with two degrees of freedom. Commun. Math. Phys. 82, 523-544 (1982)

16. Keller, J : Private communication, 1991

17. Kevrekidis, Y., McLaughlin, D.W., Winograd, M.: Movie, 1993

18. Kovacic, G., Wiggins, S : Orbits homoclinic to resonances, with an application to chaos in a model of the forced and damped sine-Gordon equation. Physica D 57, 185-225 (1992)

19. Krichever, IM.: Perturbation theory in periodic problems for two-dimensional integrable systems. Sov. Sci. Rev. C Math. 9 (1991)

20. Li, Y.: Chaotic dynamics in the PDEs. PhD thesis, Princeton University, 1993 
21 Li, Y., McLaughlin, D.W., Wiggins, S.: Homoclinic Orbits for Perturbed NLS Pde's. In preparation, 1993

22. Magnus, W., Winkler, W : Hill's equation. New York: Interscience-Wiley 1966

23 McKean, H.P.: The sine-Gordon and sinh-Gordon equations on the circle. Commun. Pure Appl. Math. 34, 197-257 (1981)

24. McKean, H.P., Trubowitz, E.: Hill's operator and hyperelliptic function theory in the presence of infinitely many branch points. Commun. Pure Appl. Math 29, 143-336 (1976)

25. McLaughlin, D.W, Overman II, E.A., Wiggins, S., Xiong, C.: Homoclinic behavior for a 4 dimensional model of a perturbed NLS equation. Preprint, Ohio State University, 1992

26 McLaughlin, D W.: Whiskered Tori for the NLS equation. In: Important developments in soliton theory, Fokas, A., Zakharov, V. (eds.) Springer Series in Nonlinear dynamics. Berlin, Heidelberg, New York: Springer 1993

27. McLaughlin, D.W., Overman, E.: Whiskered Tori for integrable PDEs and chaotic behavior in near integrable PDEs Surveys in Appl. Math 1 (1994)

28 Muraki, D.: Private communication, 1991

29. Poschel, J., Trubowitz, E.: Inverse spectral theory New York: Academic Press 1987

30. Sattinger, D.H., Zurkowski, V.D : Gauge theory of Bäcklund transformations. Physica D 26, 225-250 (1987)

31 Schober, C M.: Numerical and analytical studies of the discrete nonlinear Schrödinger equation $\mathrm{PhD}$ thesis, University of Arizona, 1991

32 Wiggins, S.: Global bifurcations and chaos: analytical methods Berlin, Heidelberg, New York: Springer 1988

33. Wilkinson, J.H.: The algebraic eigenvalue problem. London: Clarendon Press, 1965

34 Zakharov, V.E., Shabat, A B.: Exact theory of two-dimensional self-focusing and onedimensional self-modulation of waves in nonlinear media. Sov. Phys. JETP 34(1), $62-69$ (1972)

Communicated by A. Jaffe 